\title{
POINTED HOPF ACTIONS ON FIELDS, I
}

\author{
PAVEL ETINGOF AND CHELSEA WALTON
}

\begin{abstract}
Actions of semisimple Hopf algebras $H$ over an algebraically closed field of characteristic zero on commutative domains were classified recently by the authors in [18. The answer turns out to be very simple - if the action is inner faithful, then $H$ has to be a group algebra. The present article contributes to the non-semisimple case, which is much more complicated. Namely, we study actions of finite dimensional (not necessarily semisimple) Hopf algebras on commutative domains, particularly when $H$ is pointed of finite Cartan type.

The work begins by reducing to the case where $H$ acts inner faithfully on a field; such a Hopf algebra is referred to as Galois-theoretical. We present examples of such Hopf algebras, which include the Taft algebras, $u_{q}\left(\mathfrak{s l}_{2}\right)$, and some Drinfeld twists of other small quantum groups. We also give many examples of finite dimensional Hopf algebras which are not Galois-theoretical. Classification results on finite dimensional pointed Galois-theoretical Hopf algebras of finite Cartan type will be provided in the sequel, Part II, of this study.
\end{abstract}

\section{Introduction}

Let $\mathbb{k}$ be an algebraically closed field of characteristic zero, and let an unadorned $\otimes$ denote $\otimes_{\mathbb{k}}$. This work contributes to the field of noncommutative invariant theory in the sense of studying quantum analogues of group actions on commutative $\mathbb{k}$ algebras. Here, we restrict our attention to the actions of finite quantum groups, i.e. finite dimensional Hopf algebras, as these objects and their actions on (quantum) $\mathbb{k}$-algebras have been the subject of recent research in noncommutative invariant theory, including [8], 10], 16], [18, [27, 29], 34, 35, [37. The two important classes of finite dimensional Hopf algebras $H$ are those that are semisimple (as a $\mathbb{k}$-algebra) and those that are pointed (namely, all simple $H$-comodules are 1dimensional). Moreover, we have many choices of what one could consider to be a quantum $\mathbb{k}$-algebra, but from the viewpoint of classical invariant theory and algebraic geometry, the examination of Hopf actions on commutative domains over $\mathbb{k}$ is of interest. Since the classification of semisimple Hopf actions on commutative domains over $\mathbb{k}$ is understood by work of the authors [18, the focus of this article is to classify finite dimensional non-semisimple Hopf $(H-)$ actions on commutative domains over $\mathbb{k}$, particularly when $H$ is pointed.

2010 Mathematics Subject Classification. 13B05, 16T05, 81R50.

Key words and phrases. commutative domain, field, finite Cartan type, Galois-theoretical, Hopf algebra action, pointed. 
It was announced in the latest survey article of Nicolás Andruskiewitsch [1] that the classification of finite dimensional pointed Hopf algebras $H$, in the case when $H$ has an abelian group $G$ of grouplike elements, is expected to be completed soon. In this case, $H$ is a lifting of the bosonization of a Nichols algebra $\mathfrak{B}(V)$ of diagonal type by the group algebra $\mathbb{k} G$, that is to say, $g r(H) \cong \mathfrak{B}(V) \# \mathbb{k} G$. The most extensively studied class of Nichols algebras are those of finite Cartan type; their bosonizations are variations of Lusztig's small quantum groups. Prompted by the main classification result of finite dimensional pointed Hopf algebras of finite Cartan type, provided by Andruschiewitsch-Schneider in [6], we restrict our attention to the actions of such Hopf algebras on commutative domains.

All Hopf algebra actions in this work, unless otherwise specified, are assumed inner faithful in the sense that the action does not factor through a 'smaller' Hopf algebra [Definition 2.12.

We begin our study of Hopf actions on commutative domains by reducing to the case where Hopf algebras act inner faithfully on fields [Lemma 3.1, Remark [3.2]; such Hopf algebras are referred as Galois-theoretical [Definition 3.3. A general result on Galois-theoretical Hopf algebras is as follows.

Proposition 1.1 (Propositions 3.4 and 4.35). The Galois-theoretical property is preserved under taking a Hopf subalgebra, and preserved under tensor product, but is not preserved under Hopf dual, 2-cocycle deformation (that alters multiplication), nor Drinfeld twist (that alters comultiplication).

Examples of Galois-theoretical Hopf algebras include all finite group algebras, and moreover, any semisimple Galois-theoretical Hopf algebra is a group algebra [Proposition 3.4(b)]. In contrast to this, we will see below that there are many examples of non-semisimple finite dimensional pointed Galois-theoretical Hopf algebras, particularly of finite Cartan type.

Theorem 1.2. Let $q \neq \pm 1$ be a root of unity unless stated otherwise, and let $\mathfrak{g}$ be a finite dimensional simple Lie algebra.

(1) The following are examples of Galois-theoretical finite dimensional pointed Hopf algebras of finite Cartan type.

\begin{tabular}{|c|c|c|}
\hline Galois-theoretical Hopf algebra & finite Cartan type & Reference \\
\hline Taft algebras $T(n)$ & $A_{1}$ & Prop. 4.2 \\
\hline Nichols Hopf algebras $E(n)$ & $A_{1}^{\times n}$ & Prop. 4.4 \\
\hline the book algebra $\mathbf{h}(\zeta, 1)$ & $A_{1} \times A_{1}$ & Prop. 4.7 \\
\hline the Hopf algebra $H_{81}$ of dimension 81 & $A_{2}$ & Prop. 4.8 \\
\hline$u_{q}\left(\mathfrak{s l}_{2}\right)$ & $A_{1} \times A_{1}$ & Prop. 4.10 \\
\hline$u_{q}\left(\mathfrak{g l}_{2}\right)$ & $A_{1} \times A_{1}$ & Prop. 4.13 \\
\hline Twists $u_{q}\left(\mathfrak{g l}_{n}\right)^{J^{+}}, u_{q}\left(\mathfrak{g l}_{n}\right)^{J^{-}}$for $n \geq 2$ & $A_{n-1} \times A_{n-1}$ & Prop. 4.15 \\
\hline Twists $u_{q}\left(\mathfrak{s l}_{n}\right)^{J^{+}}, u_{q}\left(\mathfrak{s l}_{n}\right)^{J^{-}}$for $n \geq 2$ & $A_{n-1} \times A_{n-1}$ & Cor. 4.17 \\
\hline Twists $u_{q}^{\geq 0}(\mathfrak{g})^{J}$ for $2^{\text {rank }(\mathfrak{g})-1}$ of such $J$ & same type as $\mathfrak{g}$ & Prop. 4.28 \\
\hline
\end{tabular}


For the last three cases, $q$ is a root of unity of odd order $m \geq 3$, with $m>3$ for type $G_{2}$. Further, $n$ is relatively prime to $m$ for the result on $u_{q}\left(\mathfrak{s l}_{n}\right)^{J^{+}}$and $u_{q}\left(\mathfrak{s l}_{n}\right)^{J^{-}}$.

(2) The following are non-examples of Galois-theoretical finite dimensional pointed Hopf algebras of finite Cartan type.

\begin{tabular}{|c|c|c|}
\hline Non-Galois-theoretical Hopf algebra & finite Cartan type & Reference \\
\hline$\overline{\text { generalized Taft algs which are not Taft algs }}$ & $A_{1}$ & $\overline{\text { Prop. } \overline{4.6}}$ \\
\hline the book algebra $\mathbf{h}(\zeta, p)$ for $p \neq 1$ & $A_{1} \times A_{1}$ & Prop. 4.7 \\
\hline $\operatorname{gr}\left(u_{q}\left(\mathfrak{s l}_{2}\right)\right)$ & $A_{1} \times A_{1}$ & Prop. 4.10 \\
\hline
\end{tabular}

This theorem will be used in Part II of this work on the classification of Galoistheoretical Hopf algebras of finite Cartan type. See Remarks 4.18 and 4.29 for a preview of these results for $u_{q}\left(\mathfrak{g l}_{n}\right), u_{q}\left(\mathfrak{s l}_{n}\right), u_{q}^{\geq 0}(\mathfrak{g})$, and their twists.

For each of the Galois-theoretical Hopf algebras $H$ in the theorem above, the module fields $L$ are analyzed in terms of their invariant subfields $L^{H}$. For instance, we have the following result.

Theorem 1.3 (Theorem 3.6). Let $H$ be a finite dimensional pointed Galois-theoretical Hopf algebra with $H$-module field $L$. Then, for the group $G=G(H)$ of grouplike elements of $H$, we have the following statements:

(a) $L^{H}=L^{G}$; and

(b) the extension $L^{H} \subset L$ is Galois with Galois group $G$.

A generalization of this result is provided for finite dimensional Hopf actions on commutative domains (Theorem 3.9) and on Azumaya algebras (Theorem 3.10).

Further, we point out that finite dimensional Galois-theoretical Hopf algebras are not necessarily pointed nor semisimple [Example 4.34.

Remark 1.4. It is interesting to consider the quasiclassical analogue of our study of finite dimensional Hopf actions on commutative domains (that are faithful in some sense). To do so, let $G$ be a Poisson algebraic group and let $X$ be an irreducible algebraic variety with zero Poisson bracket. Then, the corresponding problem is to (1) determine which of such $G$ can have a faithful Poisson action on a variety $X$ as above, and (2) classify such actions. In particular, for $G^{\prime}$ a closed subgroup of $G$, this includes the problem of classifying Poisson homogeneous spaces $X=G / G^{\prime}$ which have zero Poisson bracket. Here, the Poisson bracket on the group $G$ is not necessarily zero. See [15], 26], and [38] for further reading.

This paper is organized as follows. Background material on pointed Hopf algebras and Hopf algebra actions is provided in Section 2. This includes a discussion of Hopf algebras of finite Cartan type, of quantum groups at roots of unity, and of twists of Hopf algebras and Hopf module algebras. We define and provide preliminary results on the Galois-theoretical property in Section 3. The proof of Theorem 1.2 is established in Section 4 via Propositions 4.2, 4.4, 4.6 4.8, 4.10, 4.13 4.15, 4.28, and Corollary 4.17 


\section{BACKGROUND MATERIAL}

In this section, we provide a background discussion of pointed Hopf algebras, especially those of finite Cartan type (Section 2.1) and quantum groups at roots of unity (Section 2.2). We also discuss Hopf algebra actions on $\mathbb{k}$-algebras (Section 2.3) and Drinfeld twists of these actions (Section 2.4). Consider the notation below, which will be explained in the following discussion. Unless specified otherwise:

$\mathbb{k}=$ an algebraically closed base field of characteristic zero

$\zeta, q, \omega=$ a primitive root of unity in $\mathbb{k}$ of order $n, m$, and 3 , respectively

$H=$ a finite dimensional Hopf algebra with coproduct $\Delta$, counit $\varepsilon$, antipode $S$

$G=$ the group of grouplike elements $G(H)$ of $H$

$\widehat{G}=$ character group of $G=\left\{\alpha: G \rightarrow \mathbb{k}^{\times}\right\}$

$A=$ an $H$-module algebra over $\mathbb{k}$

$L=$ an $H$-module field containing $\mathbb{k}$

$F=$ the subfield of invariants $L^{H}$

2.1. Grouplike and skew primitive elements, and pointed Hopf algebras. Consider the following notation and terminology. A nonzero element $g \in H$ is grouplike if $\Delta(g)=g \otimes g$, and the group of grouplike elements of $H$ is denoted by $G=G(H)$. An element $x \in H$ is $\left(g, g^{\prime}\right)$-skew primitive, if for grouplike elements $g, g^{\prime}$ of $G(H)$, we have that $\Delta(x)=g \otimes x+x \otimes g^{\prime}$. The space of such elements is denoted by $P_{g, g^{\prime}}(H)$.

The coradical $H_{0}$ of a Hopf algebra $H$ is the sum of all simple subcoalgebras of $H$. The coradical filtration $\left\{H_{n}\right\}_{n \geq 0}$ of $H$ is defined inductively by

$$
H_{n}=\Delta^{-1}\left(H \otimes H_{n-1}+H_{0} \otimes H\right),
$$

where $H=\bigcup_{n \geq 0} H_{n}$.

We say that a Hopf algebra $H$ is pointed if all of its simple $H$-comodules (or equivalently, if all of its simple $H$-subcoalgebras) are 1-dimensional. When $H$ is pointed, we have that $H_{0}=\mathbb{k} G$ and $H_{1}=\mathbb{k} G+\left(\sum_{g, g^{\prime} \in G} P_{g, g^{\prime}}(H)\right)$. Although this sum is not direct, one has $H_{1} / H_{0}=\bigoplus_{g, g^{\prime} \in G} \bar{P}_{g, g^{\prime}}(H)$, where $\bar{P}_{g, g^{\prime}}(H)$ is the image of $P_{g, g^{\prime}}(H)$ in $H_{1} / H_{0}$. One can verify easily the following result.

Lemma 2.1. (a) The coradical $H_{0}$ of a Hopf algebra $H$ is the group algebra $\mathbb{k} G(H)$ if and only if $H$ is pointed.

(b) If a Hopf algebra $H$ is generated by grouplike and skew primitive elements, then $H$ is pointed.

The converse of part (b) is expected in the finite dimensional case.

Conjecture 2.2. [4, Conjecture 1.4] A finite dimensional pointed Hopf algebra over an algebraically closed field of characteristic zero is generated by grouplike and skew primitive elements.

In fact, the conjecture holds in the setting of our work. 
Theorem 2.3. [7, Theorem 4.15] Conjecture 2.2 holds when $G$ is abelian.

As a consequence, a finite dimensional pointed Hopf algebra $H$ over $\mathbb{k}$ is a lifting of a bosonization of a Nichols algebra $\mathfrak{B}(V)$ by the group $G$. In other words, $\operatorname{gr}(H) \cong \mathfrak{B}(V) \# \mathbb{k} G$ in this case. Moreover, we consider a special subclass of pointed Hopf algebras, that of finite Cartan type. Refer to [5] and [6] for further details.

Definition 2.4. Let $(V, c)$ be a finite dimensional braided vector space.

- $(V, c)$ is of diagonal type if there exists a basis $x_{1}, \ldots, x_{\theta}$ of $V$ and scalars $q_{i j} \in \mathbb{k}^{\times}$so that

$$
c\left(x_{i} \otimes x_{j}\right)=q_{i j}\left(x_{j} \otimes x_{i}\right)
$$

for all $1 \leq i, j \leq \theta$. The matrix $\left(q_{i j}\right)$ is called the braiding matrix.

- $(V, c)$ is of finite Cartan type if it is of diagonal type and

$$
q_{i i} \neq 1 \text { and } q_{i j} q_{j i}=q_{i i}^{a_{i j}}
$$

where $\left(a_{i j}\right)_{1 \leq i, j \leq \theta}$ is a Cartan matrix associated to a semisimple Lie algebra.

- The same terminology applies to a Hopf algebra $H$ when $g r(H) \cong \mathfrak{B}(V) \# \mathbb{k} G$.

Many examples of finite dimensional pointed algebras of finite Cartan type are provided throughout Section 4, refer to the tables in Theorem 1.2 for a summary.

2.2. Quantum groups at roots of unity. Let us recall facts about quantum groups at roots of unity, which are examples of pointed Hopf algebras of finite Cartan type. Consider the following notation. Let $\mathfrak{g}$ be a finite dimensional simple Lie algebra over $\mathbb{k}$ of rank $r$ with Cartan matrix $\left(a_{i j}\right)$ for $i, j=1, \ldots, r$. Let $d_{i}$, for $i=1, \ldots, r$, be relatively prime integers so that the matrix $\left(d_{i} a_{i j}\right)$ is symmetric and positive definite. Let $q$ be an indeterminate.

Now consider the following Hopf algebra.

Definition 2.6. 13] [14 25] The Hopf algebra $\mathcal{U}_{q}(\mathfrak{g})$, referred to as the DrinfeldJimbo quantum group attached to $\mathfrak{g}$, is generated over $\mathbb{k}\left[q, q^{-1}\right]$ by grouplike elements $k_{i},\left(k_{i}, 1\right)$-skew primitive elements $e_{i}$, and $\left(1, k_{i}^{-1}\right)$-skew primitive elements $f_{i}$, for $i=1, \ldots, r$, with defining relations:

$$
\begin{gathered}
k_{i} e_{j} k_{i}^{-1}=q^{d_{i} a_{i j}} e_{j}, \quad k_{i} f_{j} k_{i}^{-1}=q^{-d_{i} a_{i j}} f_{j}, \quad e_{i} f_{j}-f_{j} e_{i}=\delta_{i j} \frac{k_{i}-k_{i}^{-1}}{q^{d_{i}}-q^{-d_{i}}}, \\
k_{i} k_{j}=k_{j} k_{i}, \quad k_{i} k_{i}^{-1}=k_{i}^{-1} k_{i}=1, \\
\sum_{p=0}^{1-a_{i j}}(-1)^{p}\left[\begin{array}{c}
1-a_{i j} \\
p
\end{array}\right]_{q^{d_{i}}} e_{i}^{1-a_{i j}-p} e_{j} e_{i}^{p}=0 \quad \text { for } i \neq j, \\
\sum_{p=0}^{1-a_{i j}}(-1)^{p}\left[\begin{array}{c}
1-a_{i j} \\
p
\end{array}\right]_{q^{d_{i}}} f_{i}^{1-a_{i j}-p} f_{j} f_{i}^{p}=0 \quad \text { for } i \neq j .
\end{gathered}
$$

Here, $\left[\begin{array}{c}n \\ i\end{array}\right]_{q}=\frac{[n]_{q} !}{[i]_{q} ![n-i]_{q} !}$, where $[n]_{q}=\frac{q^{n}-q^{-n}}{q-q^{-1}}$. 
At roots of unity, the Hopf algebra $\mathcal{U}_{q}(\mathfrak{g})$ has a finite dimensional quotient, which is defined below in Definition 2.9. To proceed, we must define root vectors, which was done by Lusztig using the braid group action on $\mathcal{U}_{q}(\mathfrak{g})$ [31, Theorems 3.2, 6.6(ii) and Section 4.1]. Fix a reduced decomposition $S$ of the maximal element $w_{0}$ of the Weyl group of $W: w_{0}=s_{i_{\ell}} \ldots s_{i_{1}}$. To this decomposition, there corresponds a normal ordering of positive roots: $\alpha_{S}^{(1)}=\alpha_{i_{1}}, \alpha_{S}^{(2)}=s_{i_{1}}\left(\alpha_{i_{2}}\right), \ldots$, $\alpha_{S}^{\left(i_{\ell}\right)}=s_{i_{1}} \cdots s_{i_{\ell-1}}\left(\alpha_{i_{\ell}}\right)$ [39. It is known that every positive root occurs in this sequence exactly once, and a root $\alpha+\beta$ always occurs between $\alpha$ and $\beta$. So, given a positive root $\alpha$, let $N$ be the unique number such that $\alpha=\alpha_{S}^{(N)}$, and let $w_{\alpha}^{S}=s_{i_{N-1}} \cdots s_{i_{1}}$ so that $\alpha=w_{\alpha}^{S}\left(\alpha_{i_{N}}\right)$. Define the root vectors by the formula

$$
e_{\alpha}^{S}:=T_{w_{\alpha}^{S}}\left(e_{i_{N}}\right) \quad \text { and } \quad f_{\alpha}^{S}:=T_{w_{\alpha}^{S}}\left(f_{i_{N}}\right),
$$

where, for a Weyl group element $w, T_{w}$ is the corresponding element of the braid group. Here, if $\alpha=\alpha_{i}$ is a simple root, then $e_{\alpha}=e_{i}$ and $f_{\alpha}=f_{i}$.

Now we specialize to a root of unity.

(2.7) Let $q \in \mathbb{k}$ be a root of unity of odd order $m \geq 3$, with $m>3$ for type $\mathrm{G}_{2}$.

Definition 2.8. [11, Section 1.5] The Hopf algebra $\mathcal{U}_{q}(\mathfrak{g})$ specialized to a root of unity $q$ as in (2.7) is known as the Kac-de Concini quantum group of $\mathfrak{g}$.

The desired finite dimensional quotient of $\mathcal{U}_{q}(\mathfrak{g})$ is now given as follows.

Definition 2.9. 30, Section 5.7] Take $q \in \mathbb{k}$ satisfying (2.7). There exists a finite dimensional Hopf quotient of $\mathcal{U}_{q}(\mathfrak{g})$ called the small quantum group (or the Frobenius-Lusztig kernel) attached to $\mathfrak{g}$, denoted by $u_{q}(\mathfrak{g})$. Namely, $u_{q}(\mathfrak{g})=\mathcal{U}_{q}(\mathfrak{g}) / I$, where the Hopf ideal $I$ is generated by

- $k_{i}^{m}=1$, for $i=1, \ldots, r$, and

- (nilpotency relations) $\left(e_{\alpha}^{S}\right)^{m}=\left(f_{\alpha}^{S}\right)^{m}=0$, for all positive roots $\alpha$.

Even though the elements $e_{\alpha}^{S}, f_{\alpha}^{S}$ depend on $S$, the ideal $I$ is independent of the choice of $S$ [31, Theorem 3.2]. It is also known that the elements $\left(e_{\alpha}^{S}\right)^{m}$ and $\left(f_{\alpha}^{S}\right)^{m}$, along with $k_{i}^{m}$, are central in $\mathcal{U}_{q}(\mathfrak{g})$ [11, Corollary 3.1(a)]. The Hopf algebra $u_{q}(\mathfrak{g})$ is a finite dimensional pointed Hopf algebra of dimension $m^{\operatorname{dim} \mathfrak{g}}$.

The Hopf algebra $u_{q}(\mathfrak{g})$ has Hopf subalgebras $u_{\bar{q}}^{\geq 0}(\mathfrak{g}), u_{\bar{q}}^{\leq 0}(\mathfrak{g})$, generated by the $k_{i}, e_{i}$ and the $k_{i}, f_{i}$, respectively, and subalgebras $u_{q}^{+}(\mathfrak{g}), u_{q}^{-}(\mathfrak{g})$, generated by the $e_{i}$ and by the $f_{i}$, respectively. They are quotients of the corresponding subalgebras $\mathcal{U}_{q}^{\geq 0}(\mathfrak{g}), \mathcal{U}_{q}^{\leq 0}(\mathfrak{g}), \mathcal{U}_{q}^{+}(\mathfrak{g}), \mathcal{U}_{q}^{-}(\mathfrak{g})$ of $\mathcal{U}_{q}(\mathfrak{g})$, respectively.

Remark 2.10. 31] It is known that $u_{q}(\mathfrak{g})$ is the finite dimensional Hopf subalgebra generated by $e_{i}, f_{i}, k_{i}$ inside Lusztig's "big" quantum enveloping algebra with divided powers, $U_{q}(\mathfrak{g})$, specialized to the root of unity. In fact, one has an exact sequence of Hopf algebras $u_{q}(\mathfrak{g}) \rightarrow U_{q}(\mathfrak{g}) \rightarrow U(\mathfrak{g})$, where the second map is the quantum Frobenius map [31, Section 8]. This is why $u_{q}(\mathfrak{g})$ is also referred to as the Frobenius-Lusztig kernel. 
2.3. Hopf algebra actions. We recall basic facts about Hopf algebra actions; refer to 33 for further details. A left $H$-module $M$ has a left $H$-action structure map denoted by $\cdot: H \otimes M \rightarrow M$.

Definition 2.11. Given a Hopf algebra $H$ and an algebra $A$, we say that $H$ acts on $A$ (from the left) if $A$ is a left $H$-module, $h \cdot(a b)=\sum\left(h_{1} \cdot a\right)\left(h_{2} \cdot b\right)$, and $h \cdot 1_{A}=\varepsilon(h) 1_{A}$ for all $h \in H, a, b \in A$. Here, $\Delta(h)=\sum h_{1} \otimes h_{2}$ (Sweedler notation). In this case, we also say that $A$ is a left $H$-module algebra.

In the case that $H$ acts on a field $L$, we refer to $L$ as an $H$-module field.

We restrict ourselves to $H$-actions that do not factor through 'smaller' Hopf algebras.

Definition 2.12. Given a left $H$-module $M$, we say that $M$ is an inner faithful $H$-module if $I M \neq 0$ for every nonzero Hopf ideal $I$ of $H$. Given an action of a Hopf algebra $H$ on an algebra $A$, we say that this action is inner faithful if the left $H$-module algebra $A$ is inner faithful.

When given an $H$-action on $A$, one can always pass uniquely to an inner faithful $\bar{H}$-action on $A$, where $\bar{H}$ is some quotient Hopf algebra of $H$.

We also consider elements of $A$ invariant under the $H$-action on $A$.

Definition 2.13. Let $H$ be a Hopf algebra that acts on a $\mathbb{k}$-algebra $A$ from the left. The subalgebra of invariants for this action is given by

$$
A^{H}=\{a \in A \mid h \cdot a=\varepsilon(h) a \text { for all } h \in H\} .
$$

2.4. Twists of Hopf algebras and of $H$-module algebras. Let $J=\sum J^{1} \otimes J^{2}$ be an invertible element in $H \otimes H$. Then, $J$ is a Drinfeld twist for $H$ if

- $[(\Delta \otimes i d)(J)](J \otimes 1)=[(\operatorname{id} \otimes \Delta)(J)](1 \otimes J)$, and

- $(i d \otimes \varepsilon)(J)=(\varepsilon \otimes i d)(J)=1$.

Definition 2.14. (1) The Hopf algebra $H^{J}$ is a Drinfeld twist of $H$ with respect to $J$ if $H^{J}=H$ as an algebra and $H^{J}$ has the same counit as $H$ and coproduct and antipode given by

$$
\Delta^{J}(h)=J^{-1} \Delta(h) J \quad \text { and } \quad S^{J}(h)=Q^{-1} S(h) Q,
$$

where $Q=m(S \otimes i d) J$, for all $h \in H$.

(2) Let $A$ be a left $H$-module algebra. Then, the twisted algebra $A_{J}$ has the same underlying vector space as $A$, and for $a, b \in A$, the multiplication of $A_{J}$ is given by

$$
a *_{J} b=\sum\left(J^{1} \cdot a\right)\left(J^{2} \cdot b\right) .
$$

Note that $J^{-1}$ is a twist for $H^{J}$, and $\left(H^{J}\right)^{J^{-1}} \cong H$. Also, if $A$ is an inner faithful left $H$-module algebra, then $A_{J}$ is an inner faithful left $H^{J}$-module algebra by using the same action of $H$ on the underlying vector space of $A$, and $\left(A_{J}\right)_{J^{-1}} \cong A$ as $H$-module algebras. 
As discussed in [20, page 799], Drinfeld twists $J$ have a special form when $H=$ $\mathbb{k} G$ for $G$ finite abelian. For any $\chi \in \widehat{G}$, let $\mathbf{1}_{\chi}$ be the idempotent $\frac{1}{|G|} \sum_{g \in G} \chi\left(g^{-1}\right) g$ in $\mathbb{k} G$. Then, $J=\sum_{\chi, \psi \in \widehat{G}} \sigma_{J}(\chi, \psi) \mathbf{1}_{\chi} \otimes \mathbf{1}_{\psi}$, for $\sigma_{J}$ a two-cocycle on $\widehat{G}$ with values in $\mathbb{k}^{\times}$.

We also get an alternating bicharacter $b_{J}: \widehat{G} \times \widehat{G} \rightarrow \mathbb{k}^{\times}$arising from $J$ given by $b_{J}(\chi, \psi)=\sigma_{J}(\psi, \chi) / \sigma_{J}(\chi, \psi)$ for all $\chi, \psi \in \widehat{G}$.

Proposition 2.15. [20, pages 798-799] The assignment $J \mapsto b_{J}$ is a bijection between gauge equivalence classes of Drinfeld twists and alternating bicharacters.

Now we have the following result for twisted polynomial rings.

Theorem 2.16. [20, Theorem 3.8] Let $G$ be an abelian group and let $A=\mathbb{k}\left[z_{1}, \ldots z_{n}\right]$ be a polynomial ring with a $G$-action such that $z_{i}$ are common eigenvectors of $G$. Let $\chi_{i}$ be the character of $G$ corresponding to the $G$-action on the eigenvector $z_{i}$, that is to say, $g \cdot z_{i}=\chi_{i}(g) z_{i}$. Then, the twisted algebra $A_{J}$ has generators $z_{i}$ with defining relations:

$$
z_{i} *_{J} z_{j}=b_{J}\left(\chi_{j}, \chi_{i}\right) z_{j} *_{J} z_{i}
$$

\section{Galois-theoretical Hopf algebras}

We begin by motivating the notion of a Galois theoretical Hopf algebra, or a Hopf algebra $H$ that acts inner faithfully on a field $L$. To this end, recall that our goal is to classify inner faithful actions of certain Hopf algebras on commutative domains.

Lemma 3.1. Let $A$ be a commutative domain and $Q_{A}$ be its quotient field. Namely, $Q_{A}=A \mathcal{S}^{-1}$, for the set $\mathcal{S}$ of nonzero elements of $A$. If a finite dimensional Hopf algebra $H$ acts on $A$ inner faithfully, then the action of $H$ on $A$ extends to an inner faithful action of $H$ on $Q_{A}$.

Proof. By [37, Lemma 1.1], an inner faithful $H$-action on a commutative domain $A$ extends to an inner faithful $H$-action on the localization $A \tilde{\mathcal{S}}^{-1}=A \otimes_{A^{H}} A^{H} \tilde{\mathcal{S}}^{-1}$, for $\tilde{\mathcal{S}}$ a multiplicatively closed subset of $A^{H}$. Since $A$ is a commutative domain, we have by [37, Theorem 2.5 and Proposition 2.7] that $A$ is integral over $A^{H}$. (Here, $A$ is $H$-reduced, as $A$ is a domain.) Now, take $\mathcal{S}$ to be the set of nonzero elements of $A$, and we get that the $H$-action on $A$ extends naturally to an inner faithful $H$-action on the field of quotients $Q_{A}:=A \mathcal{S}^{-1} \cong A \otimes_{A^{H}} A^{H} \tilde{\mathcal{S}}^{-1}$.

Remark 3.2. Conversely, any inner faithful $H$-action on a field $L$ yields an inner faithful $H$-action on a finitely generated commutative domain $A$. To see this, pick a finite dimensional $H$-submodule $V$ of $L$ which generates $\operatorname{Rep} H$ as a tensor category, which exists due to inner faithfulness. Take $A$ to be generated by $V$ inside $L$. Then, $H$ acts on $A$. This shows that there is always a finitely generated domain $A \subset L$ that is $H$-stable and has an inner faithful action of $H$. 
Thus, we consider Hopf algebra actions on fields for the remainder of this work. Let us introduce the following terminology.

Definition 3.3. A Hopf algebra $H$ over $\mathbb{k}$ is said to be Galois-theoretical if it acts inner faithfully and $\mathbb{k}$-linearly on a field containing $\mathbb{k}$.

Note that if a Galois-theoretical Hopf algebra $H$, say with $H$-module field $L$, yields an $H^{*}$-Galois extension $L^{H} \subset L$, then $H$ is a group algebra. However, the Hopf actions in this work do not yield Hopf-Galois extensions in general as $H$ is noncocommutative. Basic results about Galois-theoretical Hopf algebras are collected in the proposition below.

Proposition 3.4. We have the following statements.

(a) Any finite group algebra is Galois-theoretical.

(b) Any semisimple Galois-theoretical Hopf algebra is a group algebra.

(c) The restriction of an inner faithful action of a Hopf algebra to a Hopf subalgebra is inner faithful. In particular, a Hopf subalgebra of a Galoistheoretical Hopf algebra is Galois-theoretical.

(d) Any finite dimensional Galois-theoretical Hopf algebra whose coradical is a Hopf subalgebra is pointed.

(e) If $H$ and $H^{\prime}$ are Galois-theoretical Hopf algebras, then so is $H \otimes H^{\prime}$.

(f) If $H$ is Galois-theoretical, then $\mathbb{k} S_{n} \ltimes H^{\otimes n}$ is Galois-theoretical for all $n \geq 1$.

Proof. (a) It is well known that any finite group can be realized as a Galois group of a field extension.

(b) This follows from [18, Theorem 1.3].

(c) Let $H$ act inner faithfully on a module $M$ and let $H^{\prime} \subset H$ be a Hopf subalgebra. Let $I$ be a Hopf ideal of $H^{\prime}$ annihilating $M$. Let $J=H I H$. Then, $J$ is a Hopf ideal in $H$ annihilating $M$, so $J=0$ and hence, $I=0$.

(d) The coradical $H_{0}$ of $H$ is cosemisimple, and thus, semisimple by [28]. So, $H_{0}=\mathbb{k} G(H)$ by (b) and (c). Hence, $H$ is pointed by Lemma 2.1 (a).

(e) If $H$ acts on a field $L$ inner faithfully and $H^{\prime}$ acts on a field $L^{\prime}$ inner faithfully, then $H \otimes H^{\prime}$ acts on the quotient field of $L \otimes L^{\prime}$ inner faithfully.

(f) First, we need the result below.

Lemma. We have the following statements.

(i) Let $B$ be an associative algebra over $\mathbb{k}$, and $V$ be a $B$-module containing vectors $v_{1}, \ldots, v_{n}$ linearly independent over $B$ (that is to say, $V$ contains $B^{n}$ as a submodule). Then, $V^{\otimes n}$ is a faithful module over $\mathbb{k} S_{n} \ltimes B^{\otimes n}$ (where $S_{n}$ acts on $B^{\otimes n}$ by permutation of components).

(ii) Take $B$ to be a finite dimensional associative algebra over $\mathbb{k}$. If $W$ is a faithful $B$-module and $V=W \otimes X$, with $X$ an infinite dimensional $\mathbb{k}$-vector space, then $\mathbb{k} S_{n} \ltimes B^{\otimes n}$ acts faithfully on $V^{\otimes n}$ for any $n$.

(iii) If a finite dimensional Hopf algebra $H$ acts inner faithfully on an algebra $A$, then $H$ acts faithfully on $A^{\otimes s}$ for some $s$. 
Proof of Lemma. (i) Consider the map $f: \mathbb{k} S_{n} \ltimes B^{\otimes n} \rightarrow V^{\otimes n}$ given by $f(x)=$ $x \cdot\left(v_{1} \otimes \cdots \otimes v_{n}\right)$. Since the map $b \mapsto b v_{i}$ defines an isomorphism $B \rightarrow B v_{i}$, and the sum $B v_{1}+\cdots+B v_{n}$ is direct, we see that $f$ is injective, which implies (i).

(ii) Since $W^{m}$ contains a copy of $B$ for some $m$, we have that $V$ contains $B^{n}$ for any $n$. Now statement (ii) follows from (i).

(iii) Let $K_{s} \subset H$ be the kernel of the action of $H$ on $A^{\otimes s}$. Observe that $K_{s} \supset$ $K_{s+1}$ because $A^{\otimes s}=A^{\otimes s} \otimes 1 \subset A^{\otimes s+1}$. Let $K=\bigcap_{s} K_{s}$. There is an integer $s_{0}$ such that $K=K_{s}$ for all $s \geq s_{0}$. Given $h \in K$, consider the action of $\Delta(h)$ on $A^{\otimes s} \otimes A^{\otimes t}$ for $s, t \geq s_{0}$. Since $A^{\otimes s} \otimes A^{\otimes t}$ is a faithful module over $H / K \otimes H / K$, we find that $\Delta(h) \in K \otimes H+H \otimes K$. Thus, $K$ is a bialgebra ideal of $H$, hence a Hopf ideal as $H$ is finite dimensional. Since $H$ acts on $A$ inner faithfully, this implies that $K=0$, as claimed.

Now we verify part (f) of the proposition above. Fix a commutative domain $A$ over $\mathbb{k}$ that admits an inner faithful action of $H$. Then, $H$ acts faithfully on the space $W:=A^{\otimes s}$ for some $s$ by part (iii) of the Lemma. So applying part (ii) of the Lemma to $X=\mathbb{k}\left[x_{1}, \ldots, x_{s}\right]$, we conclude that $S_{n} \ltimes H^{\otimes n}$ acts faithfully on

$$
\left(A^{\otimes s}\left[x_{1}, \ldots, x_{s}\right]\right)^{\otimes n}=\left(A[x]^{\otimes s}\right)^{\otimes n}=\left(A[x]^{\otimes n}\right)^{\otimes s} .
$$

This means that $S_{n} \ltimes H^{\otimes n}$ acts inner faithfully on the commutative domain $A[x]^{\otimes n}$, where $H$ acts trivially on $x$. Thus, $S_{n} \ltimes H^{\otimes n}$ is Galois-theoretical.

Question 3.5. (a) If a finite group $\Gamma$ acts on a Galois-theoretical Hopf algebra $H$, then is $\Gamma \ltimes H$ Galois-theoretical? If true, then this would be a generalization of Proposition $3.4(\mathrm{f})$.

(b) Is a Hopf algebra quotient of a Galois-theoretical Hopf algebra $H$ also Galoistheoretical? For example, if $c$ is a central grouplike element of $H$, is then $H /(c-1)$ Galois-theoretical? In particular, if $L$ is an inner faithful $H$-module field, is then $L^{c}$ always an inner faithful $H /(c-1)$-module field?

Along with Proposition 3.4(f), special cases of Question 3.5(a) has been addressed in Propositions 4.5 and 4.9

Now we provide a general result about invariants of pointed Hopf algebra actions on commutative domains.

Theorem 3.6. (i) Let $H$ be a finite dimensional pointed Hopf algebra over $\mathbb{k}$ with $G(H)=G$, and assume that $H$ acts on a commutative domain $A$. Then, $A^{H}=A^{G}$.

(ii) If in the situation of (i), $A=L$ is a field, and $H$ acts inner faithfully on $L$, then the field extension $L^{H}=L^{G} \subset L$ is a finite Galois extension with Galois group $G$.

Proof. (i) We prove by induction in $n$ that if $x \in H_{n}$, and $\varepsilon(x)=0$ then $x$ acts by zero on $A^{G}$, which implies the required statement. For $n=0$, this is tautological as $H_{0}=\mathbb{k} G$. So let us assume that $n>0$ and that the statement is known for $n-1$. By the Taft-Wilson theorem (see [33, Theorem 5.4.1]), we may assume without loss 
of generality that

$$
\Delta(x)=g \otimes x+x \otimes g^{\prime}+w,
$$

where $g, g^{\prime} \in G, w \in H_{n-1} \otimes H_{n-1}$ and $(\varepsilon \otimes \varepsilon)(w)=0$ (as $H_{n} / H_{n-1}$ is spanned by such elements $x$ ). Let $f_{1}, f_{2} \in A^{G}$. Using the induction assumption, we have that

$$
x \cdot\left(f_{1} f_{2}\right)=\left(g \cdot f_{1}\right)\left(x \cdot f_{2}\right)+\left(x \cdot f_{1}\right)\left(g^{\prime} \cdot f_{2}\right)+w \cdot\left(f_{1} f_{2}\right)=f_{1}\left(x \cdot f_{2}\right)+\left(x \cdot f_{1}\right) f_{2} .
$$

Thus, $x: A^{G} \rightarrow A$ is a derivation.

On the other hand, since $H$ is finite dimensional, by Skryabin's theorem (37, Theorem 6.2(iii)), $A$ is integral over the subalgebra of invariants $A^{H}$. Thus, so is $A^{G}$. Hence, the equality $\left.x\right|_{A^{G}}=0$ follows from the following well known lemma from commutative algebra.

Lemma 3.7. Let $B \subset C$ be an integral extension of commutative domains, $M$ be a torsion-free $C$-module, and suppose that $x: C \rightarrow M$ is a derivation such that $\left.x\right|_{B}=0$. Then, $x=0$.

Proof of Lemma 3.7. For $c \in C$, consider the minimal monic polynomial of $c$ over $B$,

$$
p(c)=c^{n}+b_{n-1} c^{n-1}+\cdots+b_{1} c+b_{0},
$$

with $b_{i} \in B$, which exists since $C$ is integral over $B$. Letting $x$ act on the equation $p(c)=0$, we have that

$$
\left[n c^{n-1}+(n-1) b_{n-1} c^{n-2}+\cdots+b_{1}\right](x \cdot c)=0 .
$$

The first factor of the left hand side (the derivative $p^{\prime}(c)$ ) is not equal to zero due to the minimality of $p(c)$ and the fact that $n \neq 0$ (as we are in characteristic zero). Thus, since $M$ is a torsion-free $C$-module, we have $x \cdot c=0$ for all $c \in C$, as desired.

Returning to the proof of Theorem 3.6. we see that the proof of (i) is completed by applying Lemma 3.7 to $B=A^{H}, C=A^{G}, M=A$.

(ii) This follows from (i), as clearly the group $G$ must act faithfully on $L$.

Corollary 3.8. Let $H$ be a Hopf algebra (not necessarily finite dimensional) generated by a finite group of grouplike elements $G=G(H)$ and a set of $\left(g_{i}, 1\right)$-skew primitive elements $x_{i}$ for some $g_{i} \in G$. Assume that for each $i$, the Hopf subalgebra generated by $\left\{g_{i}, x_{i}\right\}$ is finite dimensional. Then:

(i) We have that $A^{H}=A^{G}$ for any commutative domain $A$ that arises as an $H$-module algebra.

(ii) If $H$ acts inner faithfully on a field $L$, then the field extension $L^{H}=L^{G} \subset L$ is Galois with Galois group $G$.

Proof. By Theorem 3.6 $x_{i}$ acts by zero on $A^{g_{i}}$, hence on $A^{G}$. This implies both statements. 
Thus, when $H$ is Galois-theoretical and generated by grouplike and skew primitive elements, the field extensions that arise as $H$-module fields may be understood in terms of classical Galois theory. This phenomenon is illustrated in several examples in the next section, particularly when $G(H)$ is a cyclic group and $L^{H} \subset L$ is a cyclic extension.

We also have the following generalization of Theorem 3.6.

Theorem 3.9. Let $H$ be a finite dimensional Hopf algebra over $\mathbb{k}$. If $H$ acts on a commutative domain $A$, then $A^{H}=A^{H_{0}}$ (even if $H_{0}$ is not a subalgebra).

Proof. As before, we show by induction in $n$ that $x \in H_{n}$ with $\varepsilon(x)=0$ acts by zero on $A^{H_{0}}$. It is shown similarly to the Taft-Wilson theorem that $H_{n} / H_{n-1}$ is spanned by elements $x_{i i^{\prime}, C, C^{\prime}}$, where $C, C^{\prime}$ are simple subcoalgebras of $H$, and

$$
\Delta\left(x_{i i^{\prime}, C, C^{\prime}}\right)=\sum_{j} t_{i j} \otimes x_{j i^{\prime}, C, C^{\prime}}+\sum_{j^{\prime}} x_{i j^{\prime}, C, C^{\prime}} \otimes t_{j^{\prime} i^{\prime}}^{\prime}+w,
$$

where $t_{i j}$ is a basis of $C$ such that $\Delta\left(t_{i j}\right)=\sum_{k} t_{i k} \otimes t_{k j}$ and $t_{i^{\prime} j^{\prime}}^{\prime}$ is a similar basis of $C^{\prime}$. Moreover, $w \in H_{n-1} \otimes H_{n-1}$ is such that $(\varepsilon \otimes \varepsilon)(w)=0$. So without loss of generality we may assume that $x=x_{i i^{\prime}, C, C^{\prime}}$. Then by the induction assumption, $x$ is a derivation of $A^{H_{0}}$ into $A$. The rest of the proof is the same as that of Theorem 3.6. (i).

Even though this paper is about actions of Hopf algebras on commutative algebras, let us give a generalization of Theorems 3.6 and 3.9 to the noncommutative case. Namely, we provide a result for Hopf actions on Azumaya algebras. Recall that examples of Azumaya algebras include matrix algebras over commutative algebras and central simple algebras.

Theorem 3.10. Let $H$ be a finite dimensional Hopf algebra over $\mathbb{k}$.

(i) Assume that $H$ acts on an Azumaya algebra $A$ with center $Z$, where $Z$ is an integral domain. Let $Z^{H}=Z \cap A^{H}$ and $Z^{H_{0}}=Z \cap A^{H_{0}}$. Then, $Z^{H}=Z^{H_{0}}$.

(ii) If, in addition to the hypotheses of (i), $H$ is pointed, then $Z^{H}=Z^{G}$ for $G=G(H)$.

Proof. (i) As in the proof of Theorem 3.9. we get that $x$ defines a derivation from $Z^{H_{0}}$ to $A$. By [16. Theorem 3.1(ii)], $A$ is integral over $Z^{H}$. Hence, $Z^{H_{0}}$ is also integral over $Z^{H}$, (i.e., $Z^{H_{0}}$ is an algebraic field extension of $Z^{H}$ ). So the statement follows from Lemma 3.7. specialized to $B=Z^{H}, C=Z^{H_{0}}$, and $M=A$.

(ii) This follows immediately from part (i) and Lemma 2.1(a).

\section{Examples and nOn-examples of Galois-Theoretical Hopf algebras}

In this section, we study examples and non-examples of finite dimensional pointed Galois-theoretical Hopf algebras, including 
- Taft algebras $T(n)$

- Nichols Hopf algebras $E(n)$

- generalized Taft algebras $T(n, m, \alpha)$

- book algebras $\mathbf{h}(\zeta, p)$

- the 81-dimensional Hopf algebra $H_{81}$

- $u_{q}\left(\mathfrak{s l}_{2}\right)$ and $\operatorname{gr}\left(u_{q}\left(\mathfrak{s l}_{2}\right)\right)$

- $u_{q}\left(\mathfrak{g l}_{2}\right)$

- some Drinfeld twists of $u_{q}\left(\mathfrak{g l}_{n}\right), u_{q}\left(\mathfrak{s l}_{n}\right)$

- some Drinfeld twists of $u_{\bar{q}}^{\geq 0}(\mathfrak{g})$ [type $\mathrm{A}_{1}$ ]

[type $\mathrm{A}_{1}^{\times n}$ ]

[type $\mathrm{A}_{1}$ ]

[type $\mathrm{A}_{1} \times \mathrm{A}_{1}$ ]

[type $\mathrm{A}_{2}$ ]

[type $\mathrm{A}_{1} \times \mathrm{A}_{1}$ ]

[type $A_{1} \times A_{1}$ ]

[type $\mathrm{A}_{n-1} \times \mathrm{A}_{n-1}$ ]

[same type as $\mathfrak{g}$ ]
(Section 4.1), (Section 4.2),

(Section 4.3),

(Section 4.4),

(Section 4.5),

(Section 4.6),

(Section 4.7),

(Section 4.7),

(Section 4.9).

Altogether, the propositions in these sections yield a proof of Theorem [1.2 An example of a Galois-theoretical Drinfeld twist of $u_{q^{1 / 2}}\left(\mathfrak{g l}_{2}\right)$ is provided in Section4.8. We also present a finite dimensional non-pointed Galois-theoretical Hopf algebra in Section 4.10. We end with a discussion of the Galois-theoretical property of duals and twists of Hopf algebras in Section 4.11

To begin, consider the notation and the preliminary result provided below.

Notation. Let $L$ be a $\mathbb{k} \mathbb{Z}_{n}$-module field, for $\mathbb{Z}_{n}=\left\langle g \mid g^{n}=1\right\rangle$. Let $\zeta$ be a primitive $n$-th root of unity. We set $L_{(i)}:=\left\{r \in L \mid g \cdot r=\zeta^{-i} r\right\}$ for $i=0, \ldots, n-1$.

Lemma 4.1. Given an inner faithful $\mathbb{k} \mathbb{Z}_{n}$-module field $L$ as above, we have that:

(a) $L$ is $\mathbb{Z}_{n}$-graded and decomposes as a direct sum of g-eigenspaces $L_{(i)}$ with eigenvalue $\zeta^{-i}$, where $L_{(0)}=L^{\mathbb{Z}_{n}}$ and $L_{(1)} \neq 0$.

(b) For $u \in L_{(1)}$, we have that $L$ is an extension of $L^{\mathbb{Z}_{n}}$, so that

$$
L=L^{\mathbb{Z}_{n}}[u] /\left(u^{n}-v\right) .
$$

Here, $v$ is a non- $n^{\prime}$-th power in $\left(L^{\mathbb{Z}_{n}}\right)^{\times}$for any $n^{\prime}>1$ dividing $n$.

Proof. Part (a) is clear. In particular, $L_{(1)} \neq 0$ due to inner faithfulness. Part (b) follows since $t^{n}-v$ is the minimal polynomial of the element $u$.

4.1. The Taft algebras $T(n)$ are Galois-theoretical. Take $n \geq 2$ and let $\zeta$ a primitive $n$-th root of unity. Let $T(n)$ be the Taft algebra of dimension $n^{2}$, which is generated by a grouplike element $g$ and a $(g, 1)$-skew primitive element $x$, subject to relations

$$
g^{n}=1, \quad x^{n}=0, \quad g x=\zeta x g .
$$

We have that $T(n)$ acts inner faithfully on the commutative domain $\mathbb{k}[z]$ by

$$
g \cdot z=\zeta^{-1} z, \quad x \cdot z=1 .
$$

So, $T(n)$ is Galois-theoretical by Lemma 3.1. More explicitly, we can extend the action of $T(n)$ on $\mathbb{k}[z]$ to an action of $T(n)$ on $\mathbb{k}(z)$ since $T(n)$ acts trivially on $\mathbb{k}\left[z^{n}\right]$ and $\mathbb{k}(z)=\mathbb{k}[z] \otimes_{\mathbb{k}\left[z^{n}\right]} \mathbb{k}\left(z^{n}\right)$. Further, we classify all inner faithful $T(n)$-module fields below, which recovers [34, Theorem 2.5]. 
Proposition 4.2. The Taft algebras $T(n)$ are Galois-theoretical, and the fields $L$ that admit an inner faithful $T(n)$-action are precisely of the form

$$
L=F[u] /\left(u^{n}-v\right)
$$

for $F=L^{T(n)}, u \in L_{(1)}$, and $v$ a non- $n^{\prime}$-th power in $F^{\times}$, for any $n^{\prime}>1$ dividing $n$. So, $L$ is a cyclic degree $n$ Galois extension of its subfield of invariants $F$ with Galois group $\mathbb{Z}_{n}$. We also have that $g \cdot u=\zeta^{-1} u, x \cdot u=1$ and $g \cdot r_{0}=r_{0}, x \cdot r_{0}=0$ for all $r_{0} \in F$.

Proof. Let us determine the $T(n)$-module fields $L$. Since $G(T(n)) \cong \mathbb{Z}_{n}$, we can employ Lemma 4.1. Observe that $L^{\mathbb{Z}_{n}}=L^{T(n)}$ by Theorem 3.6(i); let us denote this field by $F$. Take a nonzero element $u \in L_{(1)}$. Since $g \cdot(x \cdot u)=\zeta x \cdot(g \cdot u)=x \cdot u$, we have that $x \cdot u=w \in F$. Moreover, we can replace $u$ with $w^{-1} u$ to get that $x \cdot u=1$. Also, $x \cdot r_{0}=\varepsilon(x) r_{0}=0$ for all $r_{0} \in F$. Finally, the Galois group of the extension $L^{T(n)} \subset L$ is $G(T(n))=\mathbb{Z}_{n}$ by Theorem 3.6(ii).

One can reformulate Proposition 4.2 as follows.

Proposition 4.3. Fields $L \supset \mathbb{k}$ with an inner faithful $T(n)$-action are in one-toone correspondence with fields $F \supset \mathbb{k}$ together with a non- $n^{\prime}$-th power $v \in F^{\times}$, for any $n^{\prime}>1$ dividing $n$.

Proof. Retain the notation in Lemma 4.1 and Proposition 4.2. So, we have a field $L \supset \mathbb{k}$ with an inner faithful $T(n)$-action if and only if $L=F[u] /\left(u^{n}-v\right)$, where $t^{n}-v \in F[t]$ is the minimal polynomial of $u \in L_{(1)}$ over $F=L^{\mathbb{Z}_{n}}$. For $L$ to be a field, this polynomial must be irreducible. So it remains to show that the polynomial $t^{n}-v$ is irreducible if and only if $v$ is a non- $n^{\prime}$-th power in $F^{\times}$, for any $n^{\prime}>1$ dividing $n$; see, for instance, 9, Chapter 5, Section 11.8, Example 4].

The forward direction of this claim is clear. Conversely, suppose that $v \in F^{\times}$ and an irreducible polynomial $p(t)=t^{s}+q(t)$ divides $t^{n}-v$, with $\operatorname{deg} q(t)<s<n$. The group $\mathbb{Z}_{n}$ of roots of unity of order $n$ acts on such divisors by $p(t) \mapsto \zeta^{-s} p(\zeta t)$, where $\zeta$ is any $n$-th root of unity. Clearly, the stabilizer of $p(t)$ is contained in $\mathbb{Z}_{s}$ (as the constant term of $q(t)$ is nonzero). So, it must be exactly $\mathbb{Z}_{s}$. Else, there will be more than $n / s$ distinct monic irreducible divisors of $t^{n}-v$ of degree $s$, and their product must divide $t^{n}-v$, which is a contradiction. This means that $p(t)$ cannot contain any terms other than $t^{s}$ and constant term, that is to say, $p(t)=t^{s}-f$ for $f \in F$. Hence, $n / s$ is an integer, and $f^{n / s}=v$. Thus, the reverse direction of the claim holds.

4.2. The Nichols Hopf algebras $E(n)$ are Galois-theoretical. Take $n \geq 1$. Let $E(n)$ be the Nichols Hopf algebra of dimension $2^{n+1}$, generated by a grouplike element $g$ and $(g, 1)$-skew primitive elements $x_{1}, \ldots, x_{n}$, subject to relations

$$
g^{2}=1, \quad x_{i}^{2}=0, \quad g x_{i}=-x_{i} g, \quad x_{i} x_{j}=-x_{j} x_{i} .
$$


We have that $E(n)$ acts inner faithfully on the commutative domain $\mathbb{k}[z]$ and field $\mathbb{k}(z)$ by

$$
g \cdot z=-z, \quad x_{i} \cdot z=z^{2(i-1)} .
$$

One sees this as $x_{i} \cdot z^{r}=0$ for all $i$ and $r$ even. Thus, $E(n)$ is Galois-theoretical by Lemma 3.1. By a similar argument to that in Section $4.1 \mathbb{k}(z)$ is an inner faithful $E(n)$-module field.

To determine all inner faithful $E(n)$-module fields $L$, observe that $G(E(n))=\mathbb{Z}_{2}$ and use an argument similar to that in Section 4.1 to get the following result.

Proposition 4.4. The Hopf algebras $E(n)$ are Galois-theoretical and the fields $L$ that admit an inner faithful $E(n)$-action are precisely of the form

$$
L=F[u] /\left(u^{2}-v\right)
$$

for $F=L^{E(n)}, u \in L_{(1)}$, and $v$ a nonsquare element of $F^{\times}$. So, $L$ is a quadratic Galois extension of its subfield of invariants $F$ with Galois group $\mathbb{Z}_{2}$. We have that $g \cdot u=-u, x_{i} \cdot u=w_{i} \in F$ for $\left\{w_{i}\right\}_{i=1, \ldots n}$ linearly independent over $\mathbb{k}$, and $g \cdot r_{0}=r_{0}, x \cdot r_{0}=0$ for all $r_{0} \in F$.

Proof. It suffices to establish inner faithfulness. Note that any nonzero Hopf ideal of $E(n)$ has nonzero intersection with $\operatorname{span}_{\mathbb{k}}\left(x_{1}, \ldots, x_{n}\right)$ [33, Corollary 5.4.7]. So if $\left\{w_{i}\right\}_{i=1, \ldots n}$ are linearly independent, then $\left\{x_{i}\right\}_{i=1, \ldots n}$ act by linearly independent linear transformations of $L$. Thus, the action is inner faithful.

Note that while $E(n)$ can act inner faithfully on a field, it follows from the result above that $E(n)$ cannot act faithfully on a field (and hence, on a commutative domain). Indeed, the elements $g x_{i}-x_{i}$ act necessarily by zero for all $i$.

We also have the following generalization of the proposition above.

Proposition 4.5. Retain the notation above. Let $G$ be a finite subgroup of $G L_{n}(\mathbb{k})$. Then, one can form the semi-direct product Hopf algebra $\mathbb{k} G \ltimes E(n)$, where $G L_{n}(\mathbb{k})$ acts on $E(n)$ by linear transformations of the skew primitive elements $x_{i}$ for $i=$ $1, \ldots n$. Moreover, the Hopf algebra $\mathbb{k} G \ltimes E(n)$ is Galois theoretical.

Proof. For the first statement, note that one can check directly that the ideal of relations of $E(n)$ is stable under the action of $G L_{n}(\mathbb{k})$. For the last statement, proceed as follows. Let $F=\mathbb{k}\left(w_{1}, \ldots, w_{n}\right)$, where $\left\{w_{i}\right\}$ are algebraically independent, which has an action of $G$ via the embedding of $G$ into $G L_{n}(\mathbb{k})$. Pick a non-square element $v \in\left(F^{G}\right)^{\times}$. Consider the $E(n)$-module field $L=F[u] /\left(u^{2}-v\right)$. Then, the actions of $G$ and of $E(n)$ on $L$ combine into an inner faithful action of $\mathbb{k} G \ltimes E(n)$ on $L$.

4.3. On the generalized Taft algebras $T(n, m, \alpha)$ being Galois-theoretical. Let $\alpha \in \mathbb{k}$ and let $n, m$ be positive integers so that $m$ divides $n$. Let $q$ be a primitive $m$-th root of unity. Consider the generalized Taft algebra $T(n, m, \alpha)$, which is a 
Hopf algebra generated by a grouplike element $g$ and $(g, 1)$-skew primitive element $x$, subject to the relations

$$
g^{n}=1, \quad x^{m}=\alpha\left(g^{m}-1\right), \quad g x=q x g .
$$

So, $T(n, n, 0)=T(n)$ is a Taft algebra; see Section 4.1 The Galois-theoretical property of $T(n, m, \alpha)$ is given as follows.

Proposition 4.6. A generalized Taft algebra $T(n, m, \alpha)$ is Galois-theoretical if and only if $m=n$, that is to say, if and only if $T(n, m, \alpha)$ is a Taft algebra $T(n)$.

Proof. If $m=n$, then $T(n, m, \alpha)=T(n)$, and is Galois-theoretical by Proposition 4.2 ,

On the other hand, suppose $T(n, m, \alpha)$ is Galois-theoretical with inner faithful module field $L$. Since $T(n, m, \alpha)$ is generated by grouplike and skew primitive elements, $L^{T(n, m, \alpha)}=L^{G(T(n, m, \alpha))}=L^{\mathbb{Z}_{n}}$ by Theorem 3.6 let us denote this field by $F$. Then, $\mathbb{Z}_{n}=\langle g\rangle$ acts faithfully on $L$. By Lemma 4.1, $L=\bigoplus_{i=0}^{n-1} L_{(i)}$, where we can take $g \cdot r=\zeta^{i} r$ for all $r \in L_{(i)}$, with $\zeta$ is a primitive $n$-th root of unity such that $q=\zeta^{n / m}$. We also have by Lemma 4.1 that $L_{(0)}=F$ and $L=F[u] /\left(u^{n}-v\right)$ for $u \in L_{(1)}$ and $v$ a non- $n^{\prime}$-th power in $F^{\times}$, for any $n^{\prime}>1$ dividing $n$.

By way of contradiction, suppose that $n / m=s>1$, so that $T(n, m, \alpha)$ is not a Taft algebra. Since

$$
g \cdot(x \cdot u)=q x \cdot(g \cdot u)=q \zeta x \cdot u=\zeta^{s+1} x \cdot u,
$$

we get that $x \cdot u \in L_{(s+1)}$. Now $x \cdot u=r_{0} u^{s+1}$ for some $r_{0} \in F^{\times}$. Let $[d]$ denote $\frac{1-\zeta^{d}}{1-\zeta}$. Then, $x \cdot u^{d}=[d] r_{0} u^{s+d}$. Therefore,

$$
\begin{aligned}
x^{m} \cdot u & =x^{m-1} \cdot r_{0} u^{s+1} \\
& =x^{m-2} \cdot\left(r_{0}\left(x \cdot u^{s+1}\right)\right)=x^{m-2} \cdot\left([s+1] r_{0}^{2} u^{2 s+1}\right) \\
& =x^{m-3} \cdot\left([s+1][2 s+1] r_{0}^{3} u^{3 s+1}\right) \\
& \vdots \\
& =[s+1][2 s+1] \cdots[(m-1) s+1] r_{0}^{m} u v,
\end{aligned}
$$

with $[\ell s+1] \neq 0$ for $\ell=1, \ldots, m-1$. On the other hand, $\alpha\left(g^{m}-1\right) \cdot u=\alpha\left(\zeta^{m}-1\right) u$. Using the relation $x^{m}=\alpha\left(g^{m}-1\right)$, we get that

$$
v=\frac{\alpha\left(\zeta^{m}-1\right)}{[s+1][2 s+1] \cdots[(m-1) s+1]} r_{0}^{-m} .
$$

This yields a contradiction as $v$ is a non- $m$-th power in $F^{\times}$. Thus, $m=n$ as required.

4.4. On the book algebras $\mathbf{h}(\zeta, p)$ being Galois-theoretical. Let $p<n$ be coprime positive integers with $n \geq 2$ and let $\zeta$ be a primitive $n$-th root of unity. The book algebra $\mathbf{h}(\zeta, p)$ is a Hopf algebra generated by a grouplike element $g$, a $(1, g)$-skew primitive element $x_{1}$, and a $\left(g^{p}, 1\right)$-skew primitive element $x_{2}$, subject to relations:

$$
g^{n}=1, \quad x_{1}^{n}=x_{2}^{n}=0, \quad g x_{1}=\zeta x_{1} g, \quad g x_{2}=\zeta^{p} x_{2} g, \quad x_{1} x_{2}=x_{2} x_{1} ;
$$


see [2, Introduction]. The Galois-theoretical property of $\mathbf{h}(\zeta, p)$ is given as follows.

Proposition 4.7. A book algebra $\mathbf{h}(\zeta, p)$ is Galois-theoretical if and only if $p=1$. In this case, any $\mathbf{h}(\zeta, 1)$-module field $L$ is a cyclic degree $n$ Galois extension of its subfield of invariants $L^{\mathbf{h}(\zeta, 1)}$ as in Lemma 4.1.

Proof. If $p=1$, then $\mathbf{h}(\zeta, p)$ is Galois-theoretical since it acts inner faithfully on the commutative domain $\mathbb{k}[z]$ and field $\mathbb{k}(z)$ by

$$
g \cdot z=\zeta^{-1} z, \quad x_{1} \cdot z=1, \quad x_{2} \cdot z=1 .
$$

To see inner faithfulness, note that any nonzero Hopf ideal of $\mathbf{h}(\zeta, 1)$ contains either $x_{1}$ or $x_{2}$ [33, Corollary 5.4.7].

Suppose $\mathbf{h}(\zeta, p)$ is Galois-theoretical with module field $L$. Since $\mathbf{h}(\zeta, p)$ is generated by grouplike and skew primitive elements, $L^{\mathbf{h}(\zeta, p)}=L^{G(\mathbf{h}(\zeta, p))}=L^{\mathbb{Z}_{n}}$ by Theorem 3.6 let us denote this field by $F$. Then, $\mathbb{Z}_{n}$ acts faithfully on $L$. By Lemma 4.1, $L=\bigoplus_{i=0}^{n-1} L_{(i)}$, where $g \cdot r=\zeta^{-i} r$ for all $r \in L_{(i)}$. We also have by Lemma 4.1 that $L_{(0)}=F$ and $L=F[u] /\left(u^{n}-v\right)$ for $u \in L_{(1)}$ and $v$ a non- $n^{\prime}$-th power in $F^{\times}$, for any $n^{\prime}>1$ dividing $n$.

Since $g \cdot\left(x_{1} \cdot u\right)=\zeta x_{1} \cdot(g \cdot u)=x_{1} \cdot u$, we get that $x_{1} \cdot u \in F$ and we can renormalize to assume that $x_{1} \cdot u=1$. We also get that

$$
x_{1} \cdot u^{d}=\left(1+\zeta^{-1}+\cdots+\zeta^{-(d-1)}\right) u^{d-1},
$$

for all $d \geq 1$. Moreover, $g \cdot\left(x_{2} \cdot u\right)=\zeta^{p} x_{2} \cdot(g \cdot u)=\zeta^{p-1}\left(x_{2} \cdot u\right)$, so we get that $x_{2} \cdot u \in L_{(1-p)}$. Hence, $x_{2} \cdot u=r_{0} u^{1-p}$ for $r_{0} \in F^{\times}$. Now,

$$
\begin{aligned}
0=\left(x_{1} x_{2}-x_{2} x_{1}\right) \cdot u & =x_{1} \cdot\left(r_{0} u^{1-p}\right)-x_{2} \cdot 1 \\
& =r_{0}\left(x_{1} \cdot u^{1-p}\right)=r_{0} v^{-1}\left(x_{1} \cdot u^{n+1-p}\right) \\
& =r_{0}\left(1+\zeta^{-1}+\cdots+\zeta^{-(n-p)}\right) u^{n-p} .
\end{aligned}
$$

So, $1+\zeta^{-1}+\cdots+\zeta^{-(n-p)}=0$, which implies that $p=1$.

For any $\mathbf{h}(\zeta, 1)$-module field $L$, we have that the structure of $L$ is as described in Lemma 4.1

4.5. The Hopf algebra $H_{81}$ is Galois-theoretical. Let $\omega$ be a primitive cube root of unity. Let $H_{81}$ denote the 81-dimensional Hopf algebra from [36, p. 1544]; see also [3, Theorems 3.6 and 3.7]. It is generated by a grouplike element $g$ and $(g, 1)$-skew primitive elements $x, y$, subject to relations:

$$
\begin{gathered}
g^{3}=1, \quad g x=\omega x g, \quad g y=\omega y g, \quad x^{3}=0, \quad y^{3}=0, \\
x^{2} y+x y x+y x^{2}=0, \quad y^{2} x+y x y+x y^{2}=0, \quad(x y-\omega y x)^{3}=0 .
\end{gathered}
$$

Note that the relation $(x y-\omega y x)^{3}=0$ is accidentally omitted in [36, p. 1544].

Proposition 4.8. The Hopf algebra $H_{81}$ is Galois-theoretical and the fields $L$ that admit an inner faithful $\mathrm{H}_{81}$-action are precisely of the form

$$
L=F[u] /\left(u^{3}-v\right)
$$


for $F=L^{H_{81}}, u \in L_{(1)}$, and $v$ a non-cube element of $F^{\times}$. So, $L$ is a cyclic, degree 3 Galois extension of its subfield of invariants $F$ with Galois group $\mathbb{Z}_{3}$. We have that $g \cdot u=\omega^{-1} u, x \cdot u=w_{1}, y \cdot u=w_{2} \in F$ for $w_{1}, w_{2} \in F$ linearly independent over $\mathbb{k}$. Here, $g \cdot r_{0}=r_{0}, x \cdot r_{0}=y \cdot r_{0}=0$ for all $r_{0} \in F$.

Proof. Applying Lemma 3.1, we have that $H_{81}$ is Galois-theoretical as it acts on $\mathbb{k}[z]$ inner faithfully by

$$
g \cdot z=\omega^{-1} z, \quad x \cdot z=1, \quad y \cdot z=z^{3} .
$$

(One also gets that $H_{81}$ acts inner faithfully on $\mathbb{k}(z)$ by the same action.) Indeed, it is clear that $g^{3}-1, g x-\omega x g$, and $g y-\omega y g$ act on $\mathbb{k}[z]$ by zero. For the rest of the relations, note that any monomial in $x, y$ of degree $\geq 3$ acts by zero in $\mathbb{k}[z]$. To determine $H_{81}$-module fields $L$, first observe that $G\left(H_{81}\right)=\mathbb{Z}_{3}$. By an argument similar to that in Section 4.2, the result holds.

We also have the following generalization of Proposition 4.8

Proposition 4.9. Retain the notation above. Let $G$ be a finite subgroup of $G L_{2}(\mathbb{k})$. Then one can form the semi-direct product $\mathbb{k} G \ltimes H_{81}$ where $G L_{2}(\mathbb{k})$ acts on $H_{81}$ by linear transformations of the skew primitive elements $x$ and $y$. Moreover, $\mathbb{k} G \ltimes H_{81}$ is Galois theoretical.

Proof. For the first statement, one can check that the ideal of relations of $H_{81}$ is stable under the action of $G L_{2}(\mathbb{k})$. To get the last statement, adapt the proof of Proposition 4.5

4.6. The Hopf algebra $u_{q}\left(\mathfrak{s l}_{2}\right)$ is Galois-theoretical, but $\operatorname{gr}\left(u_{q}\left(\mathfrak{s l}_{2}\right)\right)$ is not. Let $m \geq 2$ and let $q$ be a root of unity in $\mathbb{k}$ with $\operatorname{ord}\left(q^{2}\right)=m$. Consider the $m^{3}$ dimensional Hopf algebra $H_{\lambda}$, generated by a grouplike element $k$, a $(k, 1)$-skew primitive element $e$, and a $\left(1, k^{-1}\right)$-skew primitive element $f$. Let $H_{\lambda}$ have relations:

$$
\text { ef }-f e=\lambda \frac{k-k^{-1}}{q-q^{-1}}, \quad k e=q^{2} e k, \quad k f=q^{-2} f k, \quad e^{m}=f^{m}=0, \quad k^{m}=1 .
$$

Note that if $\lambda \neq 0$, then $H_{\lambda} \cong u_{q}\left(\mathfrak{s l}_{2}\right)$, and without loss of generality we can take $\lambda=1$ in this case. Otherwise, $H_{\lambda=0}$ is isomorphic to the associated graded Hopf algebra $\operatorname{gr}\left(u_{q}\left(\mathfrak{s l}_{2}\right)\right)$. Part (b) of the result below recovers [34, Corollary 3.7].

Proposition 4.10. We have the following statements.

(a) The associated graded Hopf algebra $\operatorname{gr}\left(u_{q}\left(\mathfrak{s l}_{2}\right)\right)$ is not Galois-theoretical.

(b) The Hopf algebra $u_{q}\left(\mathfrak{s l}_{2}\right)$ is Galois-theoretical and the fields $L$ that admit an inner faithful $u_{q}\left(\mathfrak{s l}_{2}\right)$-action are precisely of the form $L=F[u] /\left(u^{m}-v\right)$ for $F=L^{u_{q}\left(\mathfrak{s l}_{2}\right)}, u \in L_{(1)}$, and $v$ is a non- $m^{\prime}$-th root in $F^{\times}$, for any $m^{\prime}>1$ dividing $m$. In other words, $L$ is a cyclic degree $m$ Galois extension of its subfield of invariants $F$ with Galois group $\mathbb{Z}_{m}$. Moreover, we have that

$$
e \cdot u=1, \quad f \cdot u=-q u^{2}, \quad k \cdot u=q^{-2} u,
$$

and $e \cdot r_{0}=f \cdot r_{0}=0, k \cdot r_{0}=r_{0}$ for all $r_{0} \in F$. 
Proof. (a) Suppose that $H_{\lambda}$ is Galois-theoretical with module field $L$; we will show that $\lambda \neq 0$. The subalgebra generated by $\{k, e\}$, which is isomorphic to the Taft algebra $T(m)$, acts inner faithfully on $L$ by Proposition 3.4(c). By Lemma 4.1 and Proposition 4.2 $L=\bigoplus_{i=0}^{m-1} L_{(i)}=F[u] /\left(u^{m}-v\right)$ where $L_{(i)}=\{r \in L \mid k \cdot r=$ $\left.q^{-2 i} r\right\}$, so $L_{(0)}=L^{T(m)}=: F$ and $u \in L_{(1)}$. So for $u \in L_{(1)}$ and $r_{0} \in F$, we have that

$$
k \cdot u=q^{-2} u, \quad e \cdot u=1, \quad k \cdot r_{0}=r_{0}, \quad e \cdot r_{0}=0 .
$$

Since $k \cdot(f \cdot u)=q^{-2} f \cdot(k \cdot u)=q^{-4}(f \cdot u)$, we get that $f \cdot u=r_{0} u^{2}$ for some $r_{0} \in F^{\times}$.

Now we use the relation $e f-f e=\lambda \frac{k-k^{-1}}{q-q^{-1}}$ to verify part (a). On the one hand, we have that

$$
\begin{aligned}
(e f-f e) \cdot u & =e \cdot\left(r_{0} u^{2}\right)-f \cdot 1=r_{0}\left(e \cdot u^{2}\right) \\
& =r_{0}((k \cdot u)(e \cdot u)+(e \cdot u) u)=r_{0}\left(q^{-2}+1\right) u .
\end{aligned}
$$

On the other hand, we get that

$$
\left(\lambda \frac{k-k^{-1}}{q-q^{-1}}\right) \cdot u=\frac{\lambda}{q-q^{-1}}\left(q^{-2}-q^{2}\right) u .
$$

Thus,

$$
r_{0}=\lambda \frac{\left(q^{-2}-q^{2}\right)}{\left(q-q^{-1}\right)\left(q^{-2}+1\right)}=-\lambda q .
$$

Since $r_{0} \in F^{\times}$, we must have that $\lambda \neq 0$, as required.

(b) Here, we show that $u_{q}\left(\mathfrak{s l}_{2}\right)$ is Galois-theoretical, then we use the work in part (a) to determine the structure of its module fields. First, $u_{q}\left(\mathfrak{s l}_{2}\right)$ acts on the polynomial ring $\mathbb{k}[z]$ and the field $\mathbb{k}(z)$ by

$$
e \cdot z=1, \quad f \cdot z=-q z^{2}, \quad k \cdot z=q^{-2} z .
$$

The action is inner faithful as the skew primitive elements do not act by zero; see 33. Corollary 5.4.7]. Hence, $u_{q}\left(\mathfrak{s l}_{2}\right)$ is Galois-theoretical. Now for any $u_{q}\left(\mathfrak{s l}_{2}\right)$ module field $L$, we have that $L^{u_{q}\left(\mathfrak{s l}_{2}\right)}=L^{G\left(u_{q}\left(\mathfrak{s l}_{2}\right)\right)}=L^{\mathbb{Z}_{m}}=: F$ where $\mathbb{Z}_{m}$ acts faithfully on $L$ by Theorem 3.6. By Lemma 4.1, the structure of $L$ is as claimed and part (b) holds.

We also have a slight reformulation of Proposition 4.10(b), which will be used in the sequel of this article. Let $q$ be a primitive $m$-th root of unity. Let $K_{q}$ be the $m^{3}$-dimensional Hopf algebra generated by the grouplike element $g$ and $(g, 1)$-skew primitive elements $x$ and $y$, subject to relations:

$$
g^{m}=1, \quad x^{m}=y^{m}=0, \quad g x=q x g, \quad g y=q^{-1} y g, \quad y x-q x y=1-g^{2} .
$$

Proposition 4.11. The Hopf algebra $K_{q}$ is Galois-theoretical.

Proof. We see that $K_{q^{2}}$ is isomorphic to $u_{q}\left(\mathfrak{s l}_{2}\right)$, where we identify $g, x, y$ with $k$, $e,\left(q-q^{-1}\right) k f$, respectively. 
4.7. Galois-theoretical twists of $u_{q}\left(\mathfrak{g l}_{n}\right)$ and of $u_{q}\left(\mathfrak{s l}_{n}\right)$. In this section, let $q \in \mathbb{k}$ be a root of unity of odd order $m \geq 3$ as in (2.7), and let $n \geq 2$. Recall the definition of the Kac-De Concini quantum group $\mathcal{U}_{q}\left(\mathfrak{s} \mathfrak{l}_{n}\right)$ and the small quantum group $u_{q}\left(\mathfrak{s l}_{n}\right)$ from Section 2.2. In this subsection, we need extensions of these quantum groups associated to $\mathfrak{g l}_{n}$.

To define these extensions, we first define commuting automorphisms $g_{i}$ of $\mathcal{U}_{q}\left(\mathfrak{s l}_{n}\right)$, for $i=1, \ldots, n$, by the formulas

$$
g_{i}\left(k_{j}\right)=k_{j}, \quad g_{i}\left(e_{j}\right)=q^{\delta_{i j}-\delta_{i, j+1}} e_{j}, \quad g_{i}\left(f_{j}\right)=q^{-\delta_{i j}+\delta_{i, j+1}} f_{j} .
$$

It is easy to see we get that $g_{i} g_{i+1}^{-1}$ coincides with the inner automorphism defined by the grouplike element $k_{i}$ for each $i=1, \ldots, n-1$. Moreover, the automorphisms $g_{i}$ clearly descend to the quotient Hopf algebra $u_{q}\left(\mathfrak{s l}_{n}\right)$, where they satisfy the relations $g_{i}^{m}=1$. This prompts the following definition.

Definition 4.12. The Hopf algebra $\mathcal{U}_{q}\left(\mathfrak{g l}_{n}\right)$ is the smash product of $\mathcal{U}_{q}\left(\mathfrak{s l}_{n}\right)$ with the group $\mathbb{Z}^{n}$ generated by the $g_{i}$, modulo the relations $g_{i} g_{i+1}^{-1}=k_{i}$.

The finite dimensional Hopf algebra $u_{q}\left(\mathfrak{g l}_{n}\right)$ is the smash product of $u_{q}\left(\mathfrak{s l}_{n}\right)$ with the group $(\mathbb{Z} / m \mathbb{Z})^{n}$ generated by the $g_{i}$, modulo the relations $g_{i} g_{i+1}^{-1}=k_{i}$.

More explicitly, $u_{q}\left(\mathfrak{g l}_{n}\right)$ is the Hopf algebra generated by grouplike elements $g_{i}$ for $i=1, \ldots, n,\left(k_{j}, 1\right)$-skew primitive elements $e_{j}$, and $\left(1, k_{j}^{-1}\right)$-skew primitive elements $f_{j}$, for $k_{j}:=g_{j} g_{j+1}^{-1}$, with $j=1, \ldots, n-1$, subject to relations:

$$
\begin{array}{lrr}
g_{i} e_{j} g_{i}^{-1}=q^{\delta_{i j}-\delta_{i, j+1}} e_{j}, \quad g_{i} f_{j} g_{i}^{-1}=q^{-\delta_{i j}+\delta_{i, j+1}} f_{j}, & \\
e_{i} e_{j}=e_{j} e_{i}, & f_{i} f_{j}=f_{j} f_{i}, & (|i-j| \geq 2) \\
e_{i}^{2} e_{j}-\left(q+q^{-1}\right) e_{i} e_{j} e_{i}+e_{j} e_{i}^{2}=0, & (|i-j|=1) \\
f_{i}^{2} f_{j}-\left(q+q^{-1}\right) f_{i} f_{j} f_{i}+f_{j} f_{i}^{2}=0, & (|i-j|=1) \\
g_{i} g_{j}=g_{j} g_{i}, & e_{i} f_{j}-f_{j} e_{i}=\delta_{i j} \frac{k_{i}-k_{i}^{-1}}{q-q^{-1}}, & \\
g_{i}^{m}=1, & \left(e_{\alpha}^{S}\right)^{m}=\left(f_{\alpha}^{S}\right)^{m}=0, & \alpha>0
\end{array}
$$

where $e_{\alpha}^{S}$ and $f_{\alpha}^{S}$ are the quantum root elements attached to a reduced decomposition $S$ of the maximal element $w_{0}$ of the symmetric group, as in Section 2.2. It is easy to see that $u_{q}\left(\mathfrak{g l}_{n}\right)$ has dimension $m^{n^{2}}$.

In our first result of this section, we show that $u_{q}\left(\mathfrak{g l}_{2}\right)$ is Galois-theoretical.

Proposition 4.13. The Hopf algebra $u_{q}\left(\mathfrak{g l}_{2}\right)$ is Galois-theoretical and the fields $L$ that admit an inner faithful $u_{q}\left(\mathfrak{g l}_{2}\right)$-action are precisely of the form

$$
L=F\left[u, u^{\prime}\right] /\left(u^{m}-v, u^{\prime m}-v^{\prime}\right)
$$

for $F=L^{u_{q}\left(\mathfrak{g l}_{2}\right)}, u \in L_{(1)}$, for some $v, v^{\prime} \in F^{\times}$so that $L$ is a field. In other words, $L$ is a Galois extension of its subfield of invariants $F$ with Galois group $\mathbb{Z}_{m} \times \mathbb{Z}_{m}$.

Proof. We have that $u_{q}\left(\mathfrak{g l}_{2}\right)$ acts on the field $\mathbb{k}(z)$ by extending the action of $u_{q}\left(\mathfrak{s l}_{2}\right)$ on $\mathbb{k}(z)$ from Proposition 4.10 (b) as follows:

$$
g_{1} \cdot z=q^{-1} z, \quad g_{2} \cdot z=q z, \quad e \cdot z=1, \quad f \cdot z=-q z^{2} .
$$


The action is inner faithful as the skew primitive elements do not act by zero; see 33. Corollary 5.4.7]. Hence, $u_{q}\left(\mathfrak{g l}_{2}\right)$ is Galois-theoretical. Also by Theorem 3.6. $L^{u_{q}\left(\mathfrak{g l}_{2}\right)}=L^{\mathbb{Z}_{m} \times \mathbb{Z}_{m}}$, which implies the second statement.

To study the Galois-theoretical property of twists of $u_{q}\left(\mathfrak{g l}_{n}\right)$ and of $u_{q}\left(\mathfrak{s l}_{n}\right)$, consider the quantum polynomial algebra

$$
A_{q}=\mathbb{k}\left\langle z_{1}, \ldots, z_{n}\right\rangle /\left(z_{i} z_{j}-q z_{j} z_{i}\right)_{i<j} .
$$

By [23, Theorem 4.1], we have that $A_{q}$ is a left $\mathcal{U}_{q}\left(\mathfrak{g l}_{n}\right)$-module algebra with the following action:

$$
\begin{array}{ll}
e_{i} \cdot z_{i+1}=z_{i}, & f_{i} \cdot z_{i}=z_{i+1}, \\
e_{i} \cdot z_{j}=0, & f_{i} \cdot z_{j^{\prime}}=0,
\end{array}
$$

for $j \neq i+1, j^{\prime} \neq i$. Thus, we have the following result.

Lemma 4.14. The action of $\mathcal{U}_{q}\left(\mathfrak{g l}_{n}\right)$ on $A_{q}$ above descends to an inner faithful action of $u_{q}\left(\mathfrak{g l}_{n}\right)$ on $A_{q}$.

Proof. We have that $g_{i}^{m}-1,\left(e_{\alpha}^{S}\right)^{m},\left(f_{\alpha}^{S}\right)^{m}$ generate a Hopf ideal of $\mathcal{U}_{q}\left(\mathfrak{g l}_{n}\right)$. So, to check that this Hopf ideal acts by zero on $A_{q}$, it suffices to check that it acts by zero on the generators of $A_{q}$, in this case, $z_{i}$. It is obvious that $g_{i}^{m}$ acts as the identity on $A_{q}$. Moreover, $\left(e_{\alpha}^{S}\right)^{m}$ (resp., $\left(f_{\alpha}^{S}\right)^{m}$ ) act by zero on the generators $z_{j}$, as $\left(e_{\alpha}^{S}\right)^{m}$ (resp., $\left.\left(f_{\alpha}^{S}\right)^{m}\right)$ contains more than one copy of some $e_{i}$ (resp. some $f_{i}$ ).

Since $u_{q}\left(\mathfrak{g l}_{n}\right)$ is of finite Cartan type, and thus generated by the degree one part of its coradical filtration, the only skew primitive elements of $u_{q}\left(\mathfrak{g l}_{n}\right)$ modulo the trivial ones, up to multiplication by grouplike elements and up to scaling, are $e_{i}$ and $f_{i}$. 1 Hence, the action of $u_{q}\left(\mathfrak{g l}_{n}\right)$ on $A_{q}$ is inner faithful since any nonzero Hopf ideal of $u_{q}\left(\mathfrak{g l}_{n}\right)$ has a nonzero intersection with the span of skew primitive elements of $u_{q}\left(\mathfrak{g l}_{n}\right)$ [33, Corollary 5.4.7].

Recall the discussion in Section 2.4. Let $G=(\mathbb{Z} / m \mathbb{Z})^{n}$ be the Cartan subgroup of $u_{q}\left(\mathfrak{g l}_{n}\right)$ and let $\chi_{i} \in \widehat{G}$ be defined as $\chi_{i}\left(p_{1}, \ldots, p_{n}\right)=q^{p_{i}}$. Let $J^{+}$and $J^{-}$be Drinfeld twists of $\mathbb{k} G$ so that

$$
\sigma_{J^{+}}\left(\chi_{i}, \chi_{j}\right)=\left\{\begin{array}{ll}
q & \text { for } i>j, \\
1 & \text { for } i \leq j
\end{array} \text { and } \quad \sigma_{J^{-}}\left(\chi_{i}, \chi_{j}\right)= \begin{cases}q & \text { for } i<j, \\
1 & \text { for } i \geq j .\end{cases}\right.
$$

Note that the twist $\left(J^{ \pm}\right)^{-1}$ is gauge equivalent to $J^{\mp}$.

\footnotetext{
${ }^{1}$ Let $H=\operatorname{gr}\left(u_{q}\left(\mathfrak{g l}_{n}\right)\right)$. Then, it is known that $H^{*}$ is a Hopf subalgebra in $u_{\bar{q}}^{\geq 0}\left(\mathfrak{g l}_{n}\right) \otimes u_{q}^{\leq 0}\left(\mathfrak{g l}_{n}\right)$, so it is generated in degree 1 , i.e. by the grouplike elements and $e_{i}, f_{i}$. (This is also a special case of Theorem 2.3 as $H^{*}$ is pointed with an abelian group of grouplike elements.) This implies that any homogeneous skew-primitive element $x$ of $H$ of degree $\geq 2$ is zero. Indeed, $\langle x, a b\rangle=$ $\langle\Delta(x), a \otimes b\rangle=0$ if $a, b \in H^{*}$ are of positive degree, but any element of degree $\geq 2$ in $H^{*}$ is a linear combination of elements of the form $a b$ with $\operatorname{deg}(a), \operatorname{deg}(b) \geq 1$. Thus, any skew-primitive element in $H$ modulo trivial ones is a product of a grouplike element with $e_{i}$ or $f_{i}$ up to scaling. Hence, the same is true for $u_{q}\left(\mathfrak{g l}_{n}\right)$.
} 
Let us identify $G$ with $G(H)$ via $\left(p_{1}, \ldots, p_{n}\right) \mapsto g_{1}^{p_{1}} \ldots g_{n}^{p_{n}}$. Then, we have the following result.

Proposition 4.15. The twists $u_{q}\left(\mathfrak{g l}_{n}\right)^{J^{+}}$and $u_{q}\left(\mathfrak{g l}_{n}\right)^{J^{-}}$are Galois-theoretical.

Proof. Since $A_{q}$ is an inner faithful left $u_{q}\left(\mathfrak{g l}_{n}\right)$-module algebra, $\left(A_{q}\right)_{J^{+}}$is an inner faithful left $u_{q}\left(\mathfrak{g l}_{n}\right)^{J^{+}}$-module algebra. Now by Lemma 3.1, it suffices to show that $\left(A_{q}\right)_{J^{+}}$is a commutative domain. By Theorem 2.16 we get that $\mathbb{k}\left[z_{1}, \ldots, z_{n}\right]_{\left(J^{+}\right)^{-1}} \cong$ $A_{q}$. Thus, $\mathbb{k}\left[z_{1}, \ldots, z_{n}\right]=\left(\mathbb{k}\left[z_{1}, \ldots, z_{n}\right]_{\left(J^{+}\right)^{-1}}\right)_{J^{+}}=\left(A_{q}\right)_{J^{+}}$.

By using the map $\Phi$ that relabels indices by $i \mapsto n+1-i$, we get that $A_{q^{-1}}=$ $\Phi\left(A_{q}\right)$ is a left $u_{q}\left(\mathfrak{g l}_{n}\right)=\Phi\left(u_{q}\left(\mathfrak{g l}_{n}\right)\right)$-module algebra. Following the argument above, we get that $u_{q}\left(\mathfrak{g l}_{n}\right)^{J^{-}}$is also Galois-theoretical.

Proposition 4.15 allows us to show that some quotients of $u_{q}\left(\mathfrak{g l}_{n}\right)^{J^{ \pm}}$are also Galois-theoretical. Namely, let $C$ be the subgroup of central grouplike elements in $G=G(H)$. It is clear that an element $g=g_{1}^{p_{1}} \ldots g_{n}^{p_{n}}$ is central if and only if $p_{i}=p_{i+1}$ for all $i$, that is to say, $g:=\left(g_{1} \ldots g_{n}\right)^{t}$ for some integer $t \geq 1$. So the group $C$ is isomorphic to $\mathbb{Z} / m \mathbb{Z}$ and is generated by $c:=g_{1} \ldots g_{n}$. Now, consider the Hopf algebra $u_{q}\left(\mathfrak{g l}_{n}\right)^{[s]}:=u_{q}\left(\mathfrak{g l}_{n}\right) /\left(c^{s}-1\right)$.

Proposition 4.16. We have that $\left(u_{q}\left(\mathfrak{g l}_{n}\right)^{[s]}\right)^{J^{ \pm}}$is Galois-theoretical.

Proof. Let $L$ be the $u_{q}\left(\mathfrak{g l}_{n}\right)^{J^{ \pm}}$-module field obtained from Proposition 4.15, Let $Q=\left\{z \in L \mid c^{s} \cdot z=z\right\}$. Then, $Q$ is an $\left(u_{q}\left(\mathfrak{g l}_{n}\right)^{[s]}\right)^{J^{ \pm}}$-module field, and it is easy to check directly that the action of $\left(u_{q}\left(\mathfrak{g l}_{n}\right)^{[s]}\right)^{J^{ \pm}}$on this field is inner faithful.

The algebra $u_{q}\left(\mathfrak{s l}_{n}\right)$ has a subgroup $\mathbb{Z} / n \mathbb{Z}$ consisting of central grouplike elements, and its intersection with the group $\mathbb{Z} / m \mathbb{Z}$ generated by $c$ is $\mathbb{Z} / \operatorname{gcd}(m, n) \mathbb{Z}$. So the intersection of these subgroups is trivial if and only if $\operatorname{gcd}(m, n)=1$. Thus, $u_{q}\left(\mathfrak{g l}_{n}\right) /(c-1)=u_{q}\left(\mathfrak{s l}_{n}\right)$ for $m$ and $n$ relatively prime, and we have the following result.

Corollary 4.17. If $m$ and $n$ are relatively prime, then we have that $u_{q}\left(\mathfrak{s l}_{n}\right)^{J^{ \pm}}$are Galois-theoretical (where we abuse notation and denote by $J^{ \pm}$the images of the twists $J^{ \pm}$in the quotient).

In general, we get that $\left(u_{q}\left(\mathfrak{s l}_{n}\right) /\left(c^{s}-1\right)\right)^{J^{ \pm}}$is Galois-theoretical for $s=m / \operatorname{gcd}(m, n)$.

Remark 4.18. We will show in Part II of this work that, in contrast with the above result, untwisted $u_{q}\left(\mathfrak{s l}_{n}\right)$ is not Galois-theoretical for $n \geq 3$. We will also show that the twists $J^{+}$and $J^{-}$are the only twists $J$ coming from the Cartan subgroup that make $u_{q}\left(\mathfrak{s l}_{n}\right)^{J}$ Galois-theoretical. For $u_{q}\left(\mathfrak{g l}_{n}\right)$, the situation is similar.

4.8. A modification of $u_{q^{1 / 2}}\left(\mathfrak{g l}_{2}\right)$ that is Galois-theoretical. Let $m \geq 2$ and let $q$ be a primitive $m$-th root of unity in $\mathbb{k}$. We consider a modification $u_{q}^{\prime}\left(\mathfrak{g l}_{2}\right)$ of the Hopf algebra $u_{q^{1 / 2}}\left(\mathfrak{g l}_{2}\right)$ that is of finite Cartan type. We will see that this is a special case of the Galois-theoretical Hopf algebra $u_{q^{1 / 2}}\left(\mathfrak{g l}_{2}\right)^{J^{+}}$considered above. 
The computations below follow similarly to those in previous sections, so some details are omitted.

Definition 4.19. The $m^{4}$-dimensional Hopf algebra $u_{q}^{\prime}\left(\mathfrak{g l}_{2}\right)$ is generated by grouplike elements $\gamma_{1}, \gamma_{2}$, a $\left(\gamma_{1}, 1\right)$-skew primitive element $x_{1}$, and a $\left(\gamma_{2}, 1\right)$-skew primitive element $x_{2}$, subject to relations:

$$
\begin{gathered}
\gamma_{1}^{m}=\gamma_{2}^{m}=1, \quad \gamma_{1} \gamma_{2}=\gamma_{2} \gamma_{1}, \quad x_{1}^{m}=x_{2}^{m}=0, \quad x_{2} x_{1}-q x_{1} x_{2}=1-\gamma_{1} \gamma_{2}, \\
\gamma_{1} x_{1}=q x_{1} \gamma_{1}, \quad \gamma_{1} x_{2}=q^{-1} x_{2} \gamma_{1}, \quad \gamma_{2} x_{1}=q x_{1} \gamma_{2}, \quad \gamma_{2} x_{2}=q^{-1} x_{2} \gamma_{2} .
\end{gathered}
$$

We have the following two results.

Proposition 4.20. Let $q$ be a primitive $m$-th root of unity in $\mathbb{k}$ as in (2.7). Then, we have an isomorphism of Hopf algebras $\phi: u_{q^{2}}^{\prime}\left(\mathfrak{g l}_{2}\right) \rightarrow u_{q}\left(\mathfrak{g l}_{2}\right)^{\left(J^{+}\right)^{-1}}$ given by the formulas

$$
\phi\left(\gamma_{1}\right)=g_{1}^{2}, \quad \phi\left(\gamma_{2}\right)=g_{2}^{-2}, \quad \phi\left(x_{1}\right)=e g_{1}, \quad \phi\left(x_{2}\right)=\left(q-q^{-1}\right) g_{2}^{-1} f,
$$

where the twist $J^{+}$is defined in Section 4.7 for $n=2$.

Proof. One can check this by direct computation. Here, $G=(\mathbb{Z} / m \mathbb{Z})^{2}$ is the group of grouplike elements of $u_{q}\left(\mathfrak{g l}_{2}\right)$ and we have that $J^{+}=\sum_{\chi, \psi \in \widehat{G}} \sigma_{J^{+}}(\chi, \psi) \mathbf{1}_{\chi} \otimes \mathbf{1}_{\psi}$, where $\mathbf{1}_{\chi} e=e \mathbf{1}_{\chi-\chi_{1}+\chi_{2}}$ and $\mathbf{1}_{\chi} f=f \mathbf{1}_{\chi+\chi_{1}-\chi_{2}}$.

Proposition 4.21. The Hopf algebra $u_{q}^{\prime}\left(\mathfrak{g l}_{2}\right)$ is Galois-theoretical, and the fields $L$ that admit an inner faithful $u_{q}^{\prime}\left(\mathfrak{g l}_{2}\right)$-action are of the form

$$
L=F\left[u, u^{\prime}\right] /\left(u^{m}-v, u^{\prime m}-v^{\prime}\right)
$$

for $F=L^{u_{q}^{\prime}\left(\mathfrak{g l}_{2}\right)}$, for some $v, v^{\prime} \in F^{\times}$so that $L$ is a field. In other words, $L$ is a Galois extension of its subfield of invariants $L_{q}^{u_{q}^{\prime}\left(\mathfrak{g l}_{2}\right)}$ with Galois group $\mathbb{Z}_{m} \times \mathbb{Z}_{m}$.

Proof. We have that $u_{q}^{\prime}\left(\mathfrak{g l}_{2}\right)$ acts inner faithfully on the field $\mathbb{k}\left(z_{1}, z_{2}\right)$ by

$$
\begin{array}{llll}
\gamma_{1} \cdot z_{1}=q z_{1}, & \gamma_{1} \cdot z_{2}=z_{2}, & \gamma_{2} \cdot z_{1}=z_{1}, & \gamma_{2} \cdot z_{2}=q z_{2}, \\
x_{1} \cdot z_{1}=(1-q) z_{1}^{2} z_{2}, & x_{1} \cdot z_{2}=0, & x_{2} \cdot z_{1}=0, & x_{2} \cdot z_{2}=\frac{1}{z_{1}} .
\end{array}
$$

Hence, $u_{q}^{\prime}\left(\mathfrak{g l}_{2}\right)$ is Galois-theoretical. Also by Theorem 3.6, $L^{u_{q}^{\prime}\left(\mathfrak{g l}_{2}\right)}=L^{\mathbb{Z}_{m} \times \mathbb{Z}_{m}}$, which implies the second statement.

4.9. Galois-theoretical twists of $u_{\bar{q}}^{\geq 0}(\mathfrak{g})$. Keep the notation of Section2.2. First, let $q$ be a variable (i.e., we work over $\mathbb{k}\left[q, q^{-1}\right]$ ). Fix an orientation of edges on the Dynkin diagram of $\mathfrak{g}$, and denote the corresponding oriented diagram by $Q$.

To examine the Galois-theoretical property of twists of $u_{\bar{q}}^{\geq 0}(\mathfrak{g})$, we consider the quantum polynomial algebra

$$
A_{q, Q}:=\mathbb{k}\left\langle z_{1}, \ldots, z_{r}\right\rangle /\left(z_{i} z_{j}-q^{ \pm d_{i} a_{i j}} z_{j} z_{i}\right)_{i<j},
$$

$i=1, \ldots, r$, where $\left\{z_{i}\right\}$ correspond to the vertices of the Dynkin diagram $Q$. Here, the power of $q$ is $d_{i} a_{i j}$ if the edge $i-j$ is oriented as $i \rightarrow j$, and is $-d_{i} a_{i j}$ otherwise.

We have the following well known proposition, which can be proved directly. 
Proposition 4.22. 24 [17, Proposition 3.1] The algebra $A_{q, Q}$ is a quotient of the subalgebra $\mathcal{U}_{q}^{+}(\mathfrak{g})$ of $\mathcal{U}_{q}(\mathfrak{g})$ generated by the $\left\{e_{i}\right\}$, with the quotient map sending $e_{i}$ to $z_{i}$. Namely, $A_{q, Q} \cong \mathcal{U}_{q}^{+}(\mathfrak{g}) / I_{Q}$, where $I_{Q}=\left\langle e_{i} e_{j}-q^{ \pm d_{i} a_{i j}} e_{j} e_{i}\right\rangle_{i<j}$.

The next proposition claims that the adjoint action of $\mathcal{U}_{q}^{\geq 0}(\mathfrak{g})$ on $\mathcal{U}_{q}^{+}(\mathfrak{g})$ descends to an action on $A_{q, Q}$.

Proposition 4.23. We have that $A_{q, Q}$ is a left $\mathcal{U}_{q}^{\geq 0}(\mathfrak{g})$-module algebra, where the action is induced by the (left) adjoint action of $\mathcal{U}_{\bar{q}}^{\geq 0}(\mathfrak{g})$ on itself. In other words, $h \cdot a=\sum h_{1} a S\left(h_{2}\right)$ for $h, a \in \mathcal{U}_{q}^{\geq 0}(\mathfrak{g})$, so

$$
k_{i} \cdot e_{j}=q^{d_{i} a_{i j}} e_{j} \quad \text { and } \quad e_{i} \cdot e_{j}=-q^{d_{i} a_{i j}} e_{j} e_{i}+e_{i} e_{j} .
$$

Proof. To verify the claim, it suffices to show that the ideal $I_{Q}$ is $\mathcal{U}_{q}^{+}(\mathfrak{g})$-stable under the adjoint action. Indeed, it is clear that the action of $k_{\ell}$ stabilizes $I_{Q}$. Note that

$$
e_{\ell} \cdot u=e_{\ell} u-k_{\ell} u k_{\ell}^{-1} e_{\ell},
$$

for any $u \in \mathcal{U}_{q}^{+}(\mathfrak{g})$. So, any two sided ideal of $\mathcal{U}_{q}^{+}(\mathfrak{g})$ stable under the adjoint actions of $\left\{k_{\ell}\right\}$ is also stable under the action of $\left\{e_{\ell}\right\}$, and we are done.

Now let us specialize $q$ to a root of unity of order $m$ as in (2.7) of Section 2.2 Moreover, let $C$ be the subgroup of central grouplike elements of $u_{\bar{q}}^{\geq 0}(\mathfrak{g})$; it consists of elements $\prod_{i} k_{i}^{\ell_{i}}$, such that $\sum_{i} \ell_{i} d_{i} a_{i j}$ is divisible by $m$ for all $j$. Then we have the following proposition.

Proposition 4.24. Assume (2.7). The action of $\mathcal{U}_{q}^{\geq 0}(\mathfrak{g})$ on $A_{q, Q}$ descends to an action of $u_{q}^{\geq 0}(\mathfrak{g}) /(c-1)_{c \in C}$. Moreover, this action is inner faithful.

Proof. Let $J$ be the kernel of the projection $\mathcal{U}_{\bar{q}}^{\geq 0}(\mathfrak{g}) \rightarrow u_{\bar{q}}^{\geq 0}(\mathfrak{g})$, which is a Hopf ideal. We need to show that $J$ acts by zero in $A_{q, Q}$, i.e. that $k_{i}^{m}-1$ and $\left(e_{\alpha}^{S}\right)^{m}$ act by zero. Let $Z_{0}^{\geq 0} \subset \mathcal{U}_{q}^{\geq 0}(\mathfrak{g})$ be the subalgebra generated by these elements. By [12, Proposition 5.6(d)] and [11, Corollary 3.1], we have that $Z_{0}^{\geq 0}$ is a Hopf subalgebra of $\mathcal{U}_{q}^{\geq 0}(\mathfrak{g})$ generated by central elements. Hence, if $h \in Z_{0}^{\geq 0}$, then $h \cdot a=\sum h_{1} a S\left(h_{2}\right)=\sum a h_{1} S\left(h_{2}\right)=\varepsilon(h) a$, as desired. The inner faithfulness is clear, as the kernel for the action of the grouplike elements is exactly $C$, and all skew primitive elements $e_{i}$ of $u_{q}^{\geq 0}(\mathfrak{g})$ act nontrivially by the definition of the $\mathcal{U}_{q}^{\geq 0}(\mathfrak{g})$-action on $A_{q, Q}$ from Proposition 4.23 ,

Recall the discussion in Section 2.4. Let $G=(\mathbb{Z} / m \mathbb{Z})^{r}=G\left(u_{\bar{q}}^{\geq 0}(\mathfrak{g})\right)$, and let $\alpha_{i} \in \widehat{G}$ be the simple root characters defined by $\alpha_{i}\left(k_{j}\right)=q^{d_{i} a_{i j}}$. Assume that

$$
m=\operatorname{ord}(q) \text { of (2.7) is relatively prime to } \operatorname{det}\left(a_{i j}\right) \text {, and to } 3 \text { in type } G_{2} \text {. }
$$


In this case, $C=\{1\}$, and $\alpha_{i}$ are independent generators of $\widehat{G}$. Thus, there is a unique, up to gauge transformations, Drinfeld twist $J_{Q}$ of $\mathbb{k} G$ such that

$$
b_{J_{Q}}\left(\alpha_{i}, \alpha_{j}\right)= \begin{cases}q^{d_{i} a_{i j}} & \text { for } i \rightarrow j \text { in } Q, \\ q^{-d_{i} a_{i j}} & \text { for } i \leftarrow j \text { in } Q, \\ 1 & \text { for } i \text { not connected to } j \text { in } Q .\end{cases}
$$

To see this, recall Proposition 2.15. For example, one may take the twist $J_{Q}$ defined by

$$
\sigma_{J_{Q}}\left(\alpha_{j}, \alpha_{i}\right)= \begin{cases}q^{d_{i} a_{i j}} & \text { for } i \rightarrow j \text { in } Q, \\ 1 & \text { for } i \leftarrow j \text { in } Q, \\ 1 & \text { for } i \text { not connected to } j \text { in } Q\end{cases}
$$

So we have $2^{r-1}$ such twists, up to gauge transformations. Namely, they are parametrized by orientations of the Dynkin diagram, which has $r-1$ edges, where $r=\operatorname{rank}(\mathfrak{g})$. Then, we have the following result.

Proposition 4.28. Assume (4.25). Then, the twists $u_{\bar{q}}^{\geq 0}(\mathfrak{g})^{J}$ are Galois-theoretical for each $J=J_{Q}$ as in (4.26).

Proof. By Proposition 4.24 and under the assumption of (4.25), we have that $A_{q, Q}$ is a left $u_{\bar{q}}^{\geq 0}(\mathfrak{g})$-module algebra. Hence, $\left(A_{q, Q}\right)_{J}$ is a left $u_{\bar{q}}^{\geq 0}(\mathfrak{g})^{J}$-module algebra. Now by Lemma 3.1] it suffices to show that $\left(A_{q, Q}\right)_{J}$ is a commutative domain. By Theorem [2.16, we get that $\mathbb{k}\left[z_{1}, \ldots, z_{n}\right]_{J^{-1}}=A_{q, Q}$. Thus, $\mathbb{k}\left[z_{1}, \ldots, z_{n}\right]=$ $\left(\mathbb{k}\left[z_{1}, \ldots, z_{n}\right]_{J-1}\right)_{J}=\left(A_{q, Q}\right)_{J}$

Remark 4.29. We will show in Part II of this work that the twists $J_{Q}$ above are the only ones coming from the Cartan subgroup of $u_{\bar{q}}^{\geq 0}(\mathfrak{g})$ so that $u_{\bar{q}}^{\geq 0}(\mathfrak{g})^{J_{Q}}$ is Galois-theoretical. In particular, the Hopf algebra $u_{\bar{q}}^{\geq 0}(\mathfrak{g})$ is not Galois-theoretical, unless $\mathfrak{g}=\mathfrak{s l}_{2}$. We will also see in Part II that the full small quantum group $u_{q}(\mathfrak{g})$ does not become Galois-theoretical under twists coming from the Cartan subgroup, unless $\mathfrak{g}=\mathfrak{s l}_{n}$.

If we are not in the setting of (4.25), that is to say, if $m$ is not relatively prime to the determinant of the Cartan matrix (or to 3 for type $G_{2}$ ), then the twists $J_{Q}$ as above do not exist in general. Indeed, consider the case of type $A_{n-1}$. Then $\operatorname{det}\left(a_{i j}\right)=n$ and $d_{i}=1$. Let $\omega_{i} \in \widehat{G}$ be such that $\omega_{i}\left(k_{j}\right)=q^{\delta_{i j}}$, so that $\alpha_{i}=\prod_{k} \omega_{k}^{a_{i k}}$. Let $b_{J}\left(\omega_{k}, \alpha_{j}\right)=c_{k j}$. Then from (4.26), we get that:

$$
\begin{gathered}
\prod_{k} c_{k j}^{a_{i k}}=q^{a_{i j}}, \text { if } i \rightarrow j, \quad \prod_{k} c_{k j}^{a_{i k}}=q^{-a_{i j}}, \text { if } i \leftarrow j, \\
\prod_{k} c_{k j}^{a_{i k}}=1, \text { if } i \text { not connected to } j .
\end{gathered}
$$


Recall that $c_{k j}$ are $m$-th roots of 1 ; so let $c_{i j}=q^{b_{i j}}, b_{i j} \in \mathbb{Z} / m \mathbb{Z}$. Then, we get that:

$$
\begin{gathered}
\sum_{k} a_{i k} b_{k j}=a_{i j}, \text { if } i \rightarrow j, \quad \sum_{k} a_{i k} b_{k j}=-a_{i j}, \text { if } i \leftarrow j, \\
\sum_{k} a_{i k} b_{k j}=0, \text { if } i \text { not connected to } j
\end{gathered}
$$

in $\mathbb{Z} / m \mathbb{Z}$.

Assume that $\operatorname{gcd}(m, n)=d$. The equations above yield

$$
\sum_{k=1}^{n-1} a_{i k} b_{k j}=s_{i j} a_{i j} \bmod m
$$

where $s_{i j}$ equals 1 if $i \rightarrow j$, equals -1 if $i \leftarrow j$, and equals 0 if $i$ is not connected to $j$. We also have that $\sum_{i=1}^{n-1} i a_{i k}=0 \bmod n$. Hence, $\sum_{i=1}^{n-1} \frac{i m}{d} a_{i k}=0 \bmod m$. (Indeed, if $\ell$ is divisible by $n$, then $m \ell / d$ is divisible by $m n / d$, and hence by $m$.) Therefore, $\sum_{k=1}^{n-1} \sum_{i=1}^{n-1} \frac{i m}{d} a_{i k} b_{k j}=0 \bmod m$. Now by (4.30), we get that

$$
\sum_{i} \frac{i m}{d} s_{i j} a_{i j}=0 \quad \bmod m \text {. }
$$

In particular, taking $j=1$, we get that $2 m / d=0 \bmod m$. So, $d$ divides 2 . Hence, we must have $d=1$, since $d$ divides $m$ which is odd by (2.7). Therefore, such a twist $J_{Q}$ does not exist.

However, this issue can be remedied by considering the following Hopf algebra.

Definition 4.31. The small quantum group of adjoint type, denoted by $\widetilde{u}_{q}(\mathfrak{g})$, is generated by $u_{q}(\mathfrak{g}) /(c-1)_{c \in C}$ and commuting grouplike elements $g_{i}$, subject to relations

$$
g_{i} e_{j} g_{i}^{-1}=q^{\delta_{i j}} e_{j}, \quad g_{i} f_{j} g_{i}^{-1}=q^{-\delta_{i j}} f_{j}, \quad g_{i}^{m}=1, \quad k_{i}=\prod_{j} g_{j}^{d_{i} a_{i j}} .
$$

It has dimension $m^{\operatorname{dim}(\mathfrak{g})}$, and is related to the adjoint group of $\mathfrak{g}$.

Now $\widetilde{u}_{q}(\mathfrak{g})$ has a Hopf subalgebra $\widetilde{u}_{\bar{q}}^{\geq 0}(\mathfrak{g})$, which acts inner faithfully on $A_{q, Q}$ (namely, the action is extended via $g_{i} \cdot z_{j}=q^{\delta_{i j}} z_{j}$ ). Let $G^{\prime}$ be the group of grouplike elements of $\widetilde{u}_{\bar{q}}^{\geq 0}(\mathfrak{g})$, and let $\alpha_{i}$ be the generators of $\widehat{G}^{\prime}$ defined by $\alpha_{i}\left(g_{j}\right)=q^{\delta_{i j}}$. Hence, the equation (4.27) for $\sigma_{J_{Q}}$ has a unique solution and we obtain the result below.

Proposition 4.32. The twists $\widetilde{u}_{\bar{q}}^{\geq 0}(\mathfrak{g})^{J_{Q}}$ are Galois-theoretical.

This provides $2^{\operatorname{rank}(\mathfrak{g})-1}$ Galois-theoretical Hopf algebras, without assuming the condition (4.25) on $m$.

Remark 4.33. We thank Milen Yakimov for the following remark that, in fact, there is a different way to construct the $u_{\bar{q}}^{\geq 0}(\mathfrak{g})$-module algebras $A_{q, Q}$. Namely, $A_{q, Q}$ arises as a coideal subalgebra of $\mathcal{U}_{q}^{\geq 0}(\mathfrak{g})$, and since $u_{\bar{q}}^{\geq 0}(\mathfrak{g})$ is self-dual, $A_{q, Q}$ also arises as a $u_{\bar{q}}^{\geq 0}(\mathfrak{g})$-module algebra. We see this as follows. 
There are general classification results for coideal subalgebras in $\mathcal{U}_{\bar{q}}^{\geq 0}(\mathfrak{g})$ by Heckenberger-Schneider 22 and by Heckenberger-Kolb 21. The results are that under certain natural conditions all (one-sided) coideal subalgebras are tensor products of the Cartan part of $\mathcal{U}_{q}^{\geq 0}(\mathfrak{g})$ with $\mathcal{U}^{+}[w]$ for $w \in W$ (the Weyl group). The second factor is a $q$-analog of $\mathcal{U}\left(\mathfrak{n}_{+} \cap w\left(\mathfrak{n}_{-}\right)\right)$.

All $\mathcal{U}^{+}[w]$ are iterated Ore extensions, which are $q$-polynomial rings if and only if $w$ has no repeating simple reflections in one (hence, in every) reduced decomposition, that is to say, if and only if the $w$ is a subexpression of a Coxeter element. Also, it is not hard to show that at roots of unity, the coaction of the quantum group on its coideal subalgebra descends to the small quantum group, and is inner faithful if and only if $w$ is a Coxeter element.

Therefore, the $\mathcal{U}^{+}[w]$ that (1) admit an inner faithful action of $u_{\bar{q}}^{\geq 0}(\mathfrak{g})$ and (2) are isomorphic to a $q$-polynomial algebras, are exactly those coming from the Coxeter elements of $W$.

To relate this construction to our construction of an inner faithful $u_{\bar{q}}^{\geq 0}(\mathfrak{g})$-module algebra, we need to define a bijection between orientations of the Dynkin diagram and Coxeter elements in $W$. Namely, an orientation of the Dynkin diagram defines a partial order on vertices, and we can extend it to a total order and write the corresponding word $s_{i_{1}} \ldots s_{i_{r}}$, which is a Coxeter element of $W$. Then, one can show that any two such total orderings give the same element of $W$. Conversely, given a Coxeter element, we can say that $i \rightarrow j$ if $s_{i}$ appears before $s_{j}$ in the word, and this defines an orientation on the Dynkin diagram. See [19, Exercise 3.2].

4.10. Non-pointed Galois-theoretical Hopf algebras. Consider the following example from [16].

Example 4.34. [16, Example 3.16] Let $n, m$ be positive integers and let $q$ be a primitive $m$-th root of unity. Consider the generalized Taft algebra $K=T(n m, m, 1)$ (from Section 4.3) generated by a grouplike element $g$ and a $(1, g)$-skew primitive element $x$, subject to relations $g^{n m}=1, x^{m}=g^{m}-1$, and $g x=q x g$. We get that $K$ coacts inner-faithfully on $\mathbb{k}(z)$ by the formula $\rho(z)=z \otimes g+1 \otimes x$. We also have that $K$ is not basic. Thus, $H=K^{*}$ is a non-pointed Galois-theoretical Hopf algebra by Lemma 3.1

4.11. On duals and twistings of Galois-theoretical Hopf algebras. We now discuss the preservation of the Galois-theoretical property under taking Hopf duals and twists. The results about twists (parts (b) and (c) below) were observed by Cesar Galindo; we thank him for allowing us to use this result.

Proposition 4.35. The Galois-theoretical property is preserved neither under (a) Hopf dual, (b) 2-cocycle deformation (that alters multiplication), nor (c) Drinfeld twist (that alters comultiplication).

Proof. (a) Consider Example 4.34 the Hopf dual of a generalized Taft algebra $T(n m, m, 1)$ is Galois-theoretical. However, $T(n m, m, 1)$ is not Galois-theoretical 
by Proposition 4.6 More simply, one could also use a group algebra of a finite non-abelian group as a counterexample by Proposition 3.4(a,b).

(b) Consider Proposition 4.10, $u_{q}\left(\mathfrak{s l}_{2}\right)$ is Galois-theoretical, yet its associated graded Hopf algebra $\operatorname{gr}\left(u_{q}\left(\mathfrak{s l}_{2}\right)\right)$ is not. Moreover, $\operatorname{gr}\left(u_{q}\left(\mathfrak{s l}_{2}\right)\right)$ is a 2-cocycle deformation of $u_{q}\left(\mathfrak{s l}_{2}\right)$ by [32, Theorem 7.8].

(c) Consider a Galois-theoretical group algebra $\mathbb{k} G$ and take a nontrivial Drinfeld twist $J$ of $\mathbb{k} G$ so that $(\mathbb{k} G)^{J}$ is noncocommutative. Note that $(\mathbb{k} G)^{J}$ is a semisimple Hopf algebra. So if $(\mathbb{k} G)^{J}$ is Galois-theoretical, then by Proposition 3.4 (b), ( $\left.\mathbb{k} G\right)^{J}$ is a group algebra, which yields a contradiction.

\section{APPENDIX}

This article has appeared in Transformation Groups, 20(4):985-1013, 2015. Here are the TG reference numbers versus the arXiv reference numbers:

Definitions 1-12 $=2.4,2.6,2.8,2.9,2.11,2.12,2.13,2.14,3.3,4.12,4.19,4.31$;

Theorems (T), Propositions (P), Lemmas (L), Corollaries (C), Conjectures ( $\mathrm{Cj})$ : $\mathrm{P} 1=1.1, \mathrm{~T} 2=1.2, \mathrm{~T} 3=1.3, \mathrm{~L} 4=2.1, \mathrm{Cj} 5=2.2, \mathrm{~T} 6=2.3, \mathrm{P} 7=2.15, \mathrm{~T} 8=2.18, \mathrm{~L} 9=3.1$, $\mathrm{P} 10=3.4, \mathrm{~T} 11=3.6, \mathrm{~L} 12=3.7, \mathrm{C} 13=3.8, \mathrm{~T} 14=3.9, \mathrm{~T} 15=3.10, \mathrm{~L} 16=4.1, \mathrm{P} 17-27=4.2-$ $4.11,4.13, \mathrm{~L} 28=4.14, \mathrm{P} 29=4.15, \mathrm{P} 30=4.16, \mathrm{C} 31=4.17, \mathrm{P} 32-39=4.20-4.24,4.28$, $4.32,4.35$;

Remarks 1-6=1.4, 2.10, 3.2, 4.18, 4.29, 4.33 ; Example 1=4.34 ; Equations 1-6=2.5, 2.7, 4.25, 4.26, 4.27, 4.30; Questions 1=3.5.

\section{ACKNOWLEDGMENTS}

We thank the anonymous referees for making several suggestions that improved the quality of this manuscript, which include a shorter proof of Proposition 4.23 We are grateful to Susan Montgomery for pointing out references [34 and 35. We thank Nicolás Andruskiewitsch and Iván Angiono for useful discussions and insightful suggestions. We also thank Cesar Galindo for his contribution to Section 4.11. and thank Milen Yakimov for supplying Remark 4.33. The authors were supported by the National Science Foundation: NSF-grants DMS-1000173, DMS-1102548, and DMS-1401207.

\section{REFERENCES}

[1] N. Andruskiewitsch. On finite-dimensional Hopf algebras (preprint). http://arxiv.org/pdf/1403.7838.

[2] N. Andruskiewitsch and H.-J. Schneider. Lifting of quantum linear spaces and pointed Hopf algebras of order $p^{3}$. J. Algebra, 209(2):658-691, 1998.

[3] N. Andruskiewitsch and H.-J. Schneider. Lifting of Nichols algebras of type $A_{2}$ and pointed Hopf algebras of order $p^{4}$. In Hopf algebras and quantum groups (Brussels, 1998), volume 209 of Lecture Notes in Pure and Appl. Math., pages 1-14. Dekker, New York, 2000.

[4] N. Andruskiewitsch and H.-J. Schneider. Finite quantum groups over abelian groups of prime exponent. Ann. Sci. École Norm. Sup. (4), 35(1):1-26, 2002. 
[5] N. Andruskiewitsch and H.-J. Schneider. Pointed Hopf algebras. In New directions in Hopf algebras, volume 43 of Math. Sci. Res. Inst. Publ., pages 1-68. Cambridge Univ. Press, Cambridge, 2002.

[6] N. Andruskiewitsch and H.-J. Schneider. On the classification of finite-dimensional pointed Hopf algebras. Ann. of Math. (2), 171(1):375-417, 2010.

[7] I. Angiono. On Nichols algebras of diagonal type. J. Reine Angew. Math., 683:189-251, 2013.

[8] V. A. Artamonov. Pointed Hopf algebras acting on quantum polynomials. J. Algebra, 259(2):323-352, 2003.

[9] N. Bourbaki. Algebra II. Chapters 4-7. Elements of Mathematics (Berlin). Springer-Verlag, Berlin, 2003. Translated from the 1981 French edition by P. M. Cohn and J. Howie, Reprint of the 1990 English edition [Springer, Berlin; MR1080964 (91h:00003)].

[10] K. Chan, C. Walton, Y. Wang, and J. J. Zhang. Hopf actions on filtered regular algebras. J. Algebra, 397(1):68-90, 2014.

[11] C. De Concini and V. G. Kac. Representations of quantum groups at roots of 1. In Operator algebras, unitary representations, enveloping algebras, and invariant theory (Paris, 1989), volume 92 of Progr. Math., pages 471-506. Birkhäuser Boston, Boston, MA, 1990.

[12] C. De Concini, V. G. Kac, and C. Procesi. Quantum coadjoint action. J. Amer. Math. Soc., 5(1):151-189, 1992.

[13] V. G. Drinfel'd. Hopf algebras and the quantum Yang-Baxter equation. Dokl. Akad. Nauk SSSR, 283(5):1060-1064, 1985.

[14] V. G. Drinfel'd. Quantum groups. In Proceedings of the International Congress of Mathematicians, Vol. 1, 2 (Berkeley, Calif., 1986), pages 798-820. Amer. Math. Soc., Providence, RI, 1987.

[15] V. G. Drinfel'd. On Poisson homogeneous spaces of Poisson-Lie groups. Teoret. Mat. Fiz., 95(2):226-227, 1993.

[16] P. Etingof. Galois bimodules and integrality of PI comodule algebras over invariants (preprint). http://arxiv.org/abs/1306.3821

[17] P. Etingof. Whittaker functions on quantum groups and $q$-deformed Toda operators. In Differential topology, infinite-dimensional Lie algebras, and applications, volume 194 of Amer. Math. Soc. Transl. Ser. 2, pages 9-25. Amer. Math. Soc., Providence, RI, 1999.

[18] P. Etingof and C. Walton. Semisimple Hopf actions on commutative domains. Adv. Math., 251:47-61, 2014.

[19] M. Geck and G. Pfeiffer. Characters of finite Coxeter groups and Iwahori-Hecke algebras, volume 21 of London Mathematical Society Monographs. New Series. The Clarendon Press Oxford University Press, New York, 2000.

[20] P. Guillot, C. Kassel, and A. Masuoka. Twisting algebras using non-commutative torsors: explicit computations. Math. Z., 271(3-4):789-818, 2012.

[21] I. Heckenberger and S. Kolb. Right coideal subalgebras of the Borel part of a quantized enveloping algebra. Int. Math. Res. Not. IMRN, (2):419-451, 2011.

[22] I. Heckenberger and H.-J. Schneider. Right coideal subalgebras of Nichols algebras and the Duflo order on the Weyl groupoid. Israel J. Math., 197(1):139-187, 2013.

[23] N. Hu. Quantum divided power algebra, $q$-derivatives, and some new quantum groups. $J$. Algebra, 232(2):507-540, 2000.

[24] K. Iohara and F. Malikov. Rings of skew polynomials and Gel'fand-Kirillov conjecture for quantum groups. Comm. Math. Phys., 164(2):217-237, 1994.

[25] M. Jimbo. A $q$-difference analogue of $U(\mathfrak{g})$ and the Yang-Baxter equation. Lett. Math. Phys., 10(1):63-69, 1985.

[26] E. Karolinsky. A classification of Poisson homogeneous spaces of complex reductive PoissonLie groups. In Poisson geometry (Warsaw, 1998), volume 51 of Banach Center Publ., pages 103-108. Polish Acad. Sci., Warsaw, 2000. 
[27] E. Kirkman, J. Kuzmanovich, and J. J. Zhang. Gorenstein subrings of invariants under Hopf algebra actions. J. Algebra, 322(10):3640-3669, 2009.

[28] R. G. Larson and D. E. Radford. Finite-dimensional cosemisimple Hopf algebras in characteristic 0 are semisimple. J. Algebra, 117(2):267-289, 1988.

[29] L.-Y. Liu, Q.-S. Wu, and C. Zhu. Hopf action on Calabi-Yau algebras. In New trends in noncommutative algebra, volume 562 of Contemp. Math., pages 189-209. Amer. Math. Soc., Providence, RI, 2012.

[30] G. Lusztig. Finite-dimensional Hopf algebras arising from quantized universal enveloping algebra. J. Amer. Math. Soc., 3(1):257-296, 1990.

[31] G. Lusztig. Quantum groups at roots of 1. Geom. Dedicata, 35(1-3):89-113, 1990.

[32] A. Masuoka. Abelian and non-abelian second cohomologies of quantized enveloping algebras. J. Algebra, 320(1):1-47, 2008.

[33] S. Montgomery. Hopf algebras and their actions on rings, volume 82 of CBMS Regional Conference Series in Mathematics. Published for the Conference Board of the Mathematical Sciences, Washington, DC, 1993.

[34] S. Montgomery and H.-J. Schneider. Skew derivations of finite-dimensional algebras and actions of the double of the Taft Hopf algebra. Tsukuba J. Math., 25(2):337-358, 2001.

[35] S. Montgomery and S. P. Smith. Skew derivations and $U_{q}(\operatorname{sl}(2))$. Israel J. Math., 72(1-2):158166, 1990. Hopf algebras.

[36] W. D. Nichols. Bialgebras of type one. Comm. Algebra, 6(15):1521-1552, 1978.

[37] S. Skryabin. Invariants of finite Hopf algebras. Adv. Math., 183(2):209-239, 2004.

[38] S. Zakrzewski. Poisson homogeneous spaces. In Quantum groups (Karpacz, 1994), pages 629-639. PWN, Warsaw, 1995.

[39] D. P. Zhelobenko. Kompaktnye gruppy Li i ikh predstavleniya. Izdat. "Nauka", Moscow, 1970.

Etingof: Department of Mathematics, Massachusetts Institute of Technology, CamBRIDGe, Massachusetts 02139, USA

E-mail address: etingof@math.mit.edu

Walton: Department of Mathematics, Massachusetts Institute of Technology, CamBRIDGe, Massachusetts 02139, USA

E-mail address: notlaw@math.mit.edu 